\title{
Metaplex Networks: Influence of the Exo-Endo Structure of Complex Systems on Diffusion*
}

\author{
Ernesto Estrada ${ }^{\dagger}$ \\ Gissell Estrada-Rodriguez ${ }^{\ddagger}$ \\ Heiko Gimperlein $\stackrel{\ddagger}{\ddagger}$
}

\begin{abstract}
In a complex system, the interplay between the internal structure of its entities and their interconnection may play a fundamental role in the global functioning of the system. Here, we define the concept of metaplex, which describes such a trade-off between the internal structure of entities and their interconnections. We then define a dynamical system on a metaplex and study diffusive processes on it. We provide analytical and computational evidence on the role played by the size of the nodes, the location of the internal coupling areas, and the strength and range of the coupling between the nodes on the global dynamics of metaplexes. Finally, we extend our analysis to two real-world metaplexes: a landscape and a brain metaplex. We corroborate that the internal structure of the nodes in a metaplex can dominate the global dynamics (brain metaplex) or play a regulatory role (landscape metaplex) in the influence of the interconnection between nodes.
\end{abstract}

Key words. complex networks, metaplex network, $d$-path Laplacian, diffusion

AMS subject classifications. 05C $81,92 \mathrm{C} 99,35 \mathrm{P} 05,47 \mathrm{G} 99$

DOI. $10.1137 / 18 \mathrm{M} 1236253$

\section{Contents}

I Introduction

3 Metaplexes: Structure and Dynamics $\quad 623$

4 Operators and Spectra for Metaplexes

\footnotetext{
* Received by the editors January 2, 2019; accepted for publication (in revised form) October 9, 2019; published electronically August 6, 2020.

https://doi.org/10.1137/18M1236253

Funding: The work of the second author was supported by the Maxwell Institute Graduate School in Analysis and Its Applications, a Centre for Doctoral Training funded by the UK Engineering and Physical Sciences Research Council (grant EP/L016508/01), the Scottish Funding Council, HeriotWatt University, and the University of Edinburgh. The work of the third author was supported by ERC Advanced Grant HARG 268105.

$\dagger$ Institute of Applied Mathematics (IUMA), Universidad de Zaragoza, E-50009 Zaragoza, Spain, ARAID Foundation, Government of Aragón, E-50018 Zaragoza, Spain, and Instituto de Ciencias Matematicas e de Computacao, Universidade de Sao Paulo, 13560-970 Sao Carlos, Sao Paulo, Brazil (estrada66@unizar.es).

${ }^{\ddagger}$ Maxwell Institute for Mathematical Sciences and Department of Mathematics, Heriot-Watt University, Edinburgh, EH14 4AS, United Kingdom (ge5@hw.ac.uk, h.gimperlein@hw.ac.uk).
} 
4.2 Weakly and Strongly Interacting Limits . . . . . . . . . . . . . 626

4.3 Numerical Results of the Spectral Properties . . . . . . . . . . . 626

5 Analysis of Diffusion in Metaplexes $\quad 628$

6 Numerical Analysis of Diffusion in Metaplexes 629

6.1 Setup of Numerical Experiments . . . . . . . . . . . . . . 630

6.2 Experiment 1: Central Coupling Region . . . . . . . . . . . . . 631

6.3 Experiment 2: Distant Coupling Regions . . . . . . . . . . . . . . . 632

6.3.1 Metaplex Superdiffusion ............... 634

7 Real-World Metaplexes $\quad 634$

7.1 Landscape Metaplex . . . . . . . . . . . . . . . . . . . . . 636

7.2 Visual Cortex Metaplex of Macaque . . . . . . . . . . . . . . . . 640

8 Summary $\quad 642$

$\begin{array}{ll}\text { References } & 643\end{array}$

I. Introduction. A complex system is characterized by the existence of many components which are interdependent on one another [20, 40, 49]. Each of these components is at the same time characterized by a certain structure, dynamics, and function [4], which influence the global behavior of the system. The interconnection between these components represents the exoskeleton of the complex system, and it is well characterized by the use of complex networks [20, 40, 49]. The internal structure of the corresponding entities - inside the nodes of the complex networkrepresents the endostructure of the system and is not necessarily a network in itself. Next we consider some examples, selected from the vast variety that exists in nature and society, to illustrate this point.

The first type of system, represented in Figure 1(a), is formed by cells that are interconnected by means of their physical contacts, such as in the case of cellular systems in tissues, neuronal networks, or astrocytic complexes, i.e., star-shaped glial cells [48]. In this case the exoskeleton of the system is the cellular network per se, and the endoskeleton is described by the crowded environment inside the cells, in which up to $40 \%$ of the cytoplasmic volume is occupied by RNA, ribosomes, and proteins $[69,70]$. The situation is similar if we consider regions inside an organ instead of individual cells. A typical example is that of anatomical or functional regions of the brain [8]. Here the system is again characterized by an exoskeleton formed by the brain network and an endostructure describing the interior of those regions.

In the second example, illustrated in Figure 1(b), we consider an enriched conceptual metaplex. This is a conceptual organization model inferred by Goñi et al. [31] from verbal fluency of 200 individuals. The study was aimed at finding the conceptual storage structure of the natural category of animals as a network. Thus, every node of the metaplex represents a category of animals, e.g., pets, and inside the nodes we find all the words in that category.

Our third example consists of the patches formed in an ecological landscape as illustrated in Figure 1(c). In this case, the system of patches and the corridors connecting them form an exoskeleton known as a landscape network [63]. The combination of the geographic and ecological features of the individual patches determines their endostructures. This example will be discussed in what follows. 


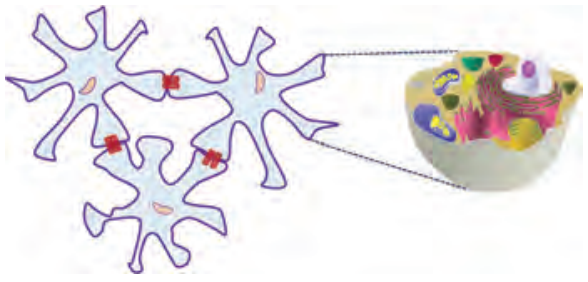

(a)

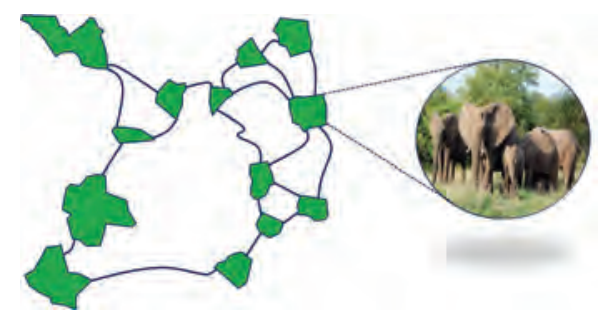

(c)

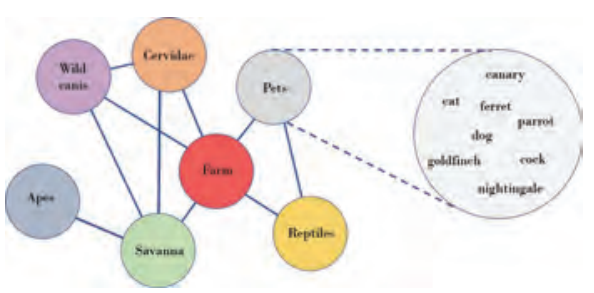

(b)

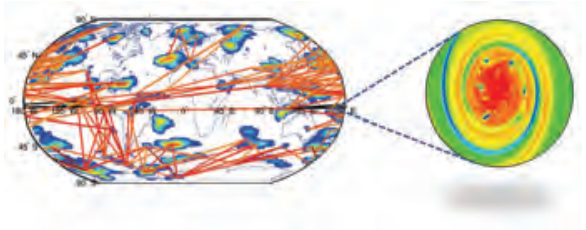

(d)

Fig. I (a) Illustration of a cellular system formed by biological cells connected by means of gap junctions to interchange chemicals and a zoom of the internal structure of a cell. (b) Enriched conceptual system representing the conceptual organization of animal categories. (c) Landscape ecological system formed by patches interconnected by corridors used by some species to move from patch to patch. Zooming in reveals the foraging movement of these species inside the patches. (d) Climate system formed by a network of climatic event correlations and the internal climatic events at local regions.

The final example is, however, at a much larger scale (see Figure 1(d)). It corresponds to a climate system in which the nodes represent geographical regions in the world. These regions are connected by climatic correlations or causalities, such that two regions are connected if a climatic event in one region triggers a climatic event in the other $[67,15]$. Inside every region, however, there is a vast collection of complex phenomena taking place which are typically modeled using weather and local climatic models.

A common dynamical process occurring in the systems described in Figure 1 is diffusion, which is also ubiquitous in many other physical [26], chemical [38], biological, and socioeconomic systems [35, 41], especially on mesoscopic scales [68]. In the systems described above, and in many other complex systems, there is a trade-off between a diffusive process taking place inside the entities of the systems and the diffusion taking place between them. When both the endo- and the exostructures of the complex system are representable by networks, we can use some of the physicomathematical tools already available in the literature, such as networks of networks $[27,59]$ or multiplexes $[3,30,36,55]$. However, the main challenge here is that in many cases the endostructure of the system is described by a continuous space, while the exoskeleton is a discrete one, e.g., a network. In the case of cells, their interior is formed mainly by cytoplasm, which is $80 \%$ water [43], while their interconnection is well described by a cellular network. Goñi et al. [31] considered in their work the idea that inside each category every pair of words is connected. Thus, it is assumed that the internal space inside categories is a continuum in which words are found in a random-walk-like navigation inside the memory of individuals. In the next example, 
where species are moving in a patchy environment, the movement inside the patches is better described using continuous diffusive models [1, 9], and the hop between patches through the narrow corridors is better accounted for by using a network-theoretic approach [63]. Finally, in climate systems the processes inside the regions - nodes - are well described by aerodynamics and fluid dynamics, while the causal influence between regions is well described by a network of interactions, including long range effects $[15,67]$.

The implications of this endo-exo trade-off in complex systems is very important for understanding their function. There is experimental evidence, for instance, that the movement of small molecules inside a cell follows an anomalous diffusive process, either subdiffusive $[29,51,65]$ or superdiffusive [56]. The distinction between normal (or Fickian) and anomalous diffusion is made on the basis of the scaling of the mean squared displacement $\left\langle x^{2}\right\rangle=\left\langle\left(x-x_{0}\right)^{2}\right\rangle$ of the diffusive particle with time [7, 46], where $x$ is the current position of a particle and $x_{0}$ is the initial position. See the supplementary material for a more detailed description of anomalous diffusion. While for normal diffusion the mean squared displacement scales linearly with time $\left\langle x^{2}\right\rangle \sim t$, for anomalous diffusion it scales as a power law $\left\langle x^{2}\right\rangle \sim t^{\alpha}$ with exponent $\alpha \in(0,2)$ larger (super-) or smaller (sub-) than 1. This means that on long time scales, in a superdiffusive process the space explored by the diffusive particle is larger than the space explored in a normal diffusion in exactly the same time, due to the high probability of long range hopping. In the case of a small molecule inside a cell, anomalous diffusion is mainly due to the crowded environment inside the cells [58,60]. However, there are more complex mechanisms inside certain types of cells that can lead to anomalous diffusive processes. One example is the generation of calcium waves [10], which have been observed in cardiac muscle [25], skeletal muscle fiber [16], medaka eggs [57], and astrocytes [11]. This subdiffusive $\mathrm{Ca}^{2+}$ movement might be involved with cardiac $[39,42]$ and brain $[66,34]$ diseases.

When global systems, such as tissues [50] or the whole brain [8], are analyzed, superdiffusive processes can be experimentally observed for fresh specimens of carcinoma, fibrous mastopathies, adipose, and liver tissues [37] as well as for signals navigating across regions of the brain [12]. Interesting research questions emerge from these experiments: Is the anomalous diffusive behavior of a global system the consequence of sub- or superdiffusive processes inside the entities, e.g., cells or regions? Are they the result of the anomalous diffusion between the entities only? Is it the combination of the exo- and endostructures that determines the nature of the diffusive process in a complex system? How relevant is the endostructure depending on the type of network, i.e., a small-world network? Similar questions emerge for the analysis of other systems such as the landscape system discussed above. In landscape systems the superdiffusive movement of species inside a patch is well documented and described by continuous models $[1,9,64]$. Can this behavior alone determine the nature of the diffusive process at the global landscape level?

In this work we answer these questions by introducing the concept of metaplexes. Informally, a metaplex is a representation of a complex system in which the internal structure of the nodes and the interconnection between them are considered at the same time (see Figure 2). Thus, a dynamical system on a metaplex consists of the coupling between the dynamics inside the entities, typically a continuous space, and the dynamics between these entities, which is controlled by the interentity connectivity.

Our results show that in a linear metaplex, superdiffusion due to long range hopping in a network, such as in [22], survives irrespective of the internal structure of 


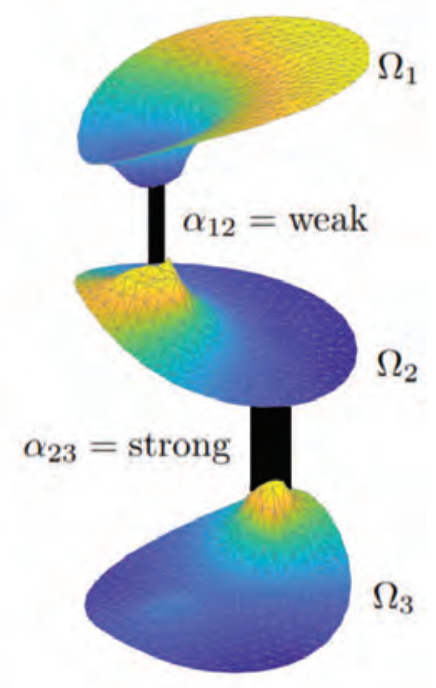

Fig. 2 Diffusion in a metaplex. The domains $\Omega_{j}$ are the nodes of the graph (exoskeleton) in which a continuous diffusion process takes place. The connections with different strengths, given by $\alpha_{12}$ and $\alpha_{23}$, account for the edges of the graph. The colors indicate the "density" of the diffusing particles, with yellow for higher concentration.

the nodes. On the other hand, we prove that superdiffusion in the nodes can speed up regular diffusion in the metaplex, but it cannot lead to superdiffusion. The geometry of the nodes and their coupling play crucial roles for the global dynamics, which we explain via a combination of analysis and numerical experiments. The results shed light on the rich and substantially different nature of the dynamics of metaplexes and the interplay of their exo- and endostructures, including in the real-world systems considered in subsection 6.3.1.

Here we start with a formal definition of a metaplex and a dynamical system on the metaplex. We then study a linear toy model that allows us to understand some of the fundamental principles of diffusive processes on metaplexes. Finally, we study two real-world metaplexes indicating the potential applications of this representation of complex systems.

2. Preliminaries. In this section we define the concepts and notation to be used in this article. Here we consider two kinds of diffusive dynamical systems, one taking place in a continuous space and the other in a discrete space. Let us start by defining the diffusive process on the continuous space.

Let $u(t, \mathbf{x})$ be the density of the diffusive particle at time $t$ for $\mathbf{x}$ in a domain in $\mathbb{R}^{n}$. The density $u(t, \mathbf{x})$ evolves according to

$$
\partial_{t} u(t, \mathbf{x})=(-\Delta)^{s} u(t, \mathbf{x}),
$$

where $(-\Delta)^{s}$ denotes a fractional Laplacian operator for $s \in(0,1]$. The application of the fractional Laplacian to $u(t, \mathbf{x})$ on $\mathbb{R}^{n}$ at a fixed time $t$ is given by

$$
(-\Delta)^{s} u(\mathbf{x})=c_{n, s} P . V . \int_{\mathbb{R}^{n}} \frac{u(\mathbf{x})-u(\mathbf{y})}{|\mathbf{x}-\mathbf{y}|^{n+2 s}} d \mathbf{y}=c_{n, s} \lim _{\varepsilon \rightarrow 0^{+}} \int_{\{|\mathbf{y}|>\varepsilon\}} \frac{u(\mathbf{x})-u(\mathbf{y})}{|\mathbf{x}-\mathbf{y}|^{n+2 s}} d \mathbf{y},
$$


where P.V. denotes the Cauchy principal value and $c_{n, s}$ is the following normalization constant in terms of Euler Gamma functions:

$$
c_{n, s}=\frac{2^{2 s} s \Gamma\left(\frac{n+2 s}{2}\right)}{\pi^{\frac{n}{2}} \Gamma(1-s)} .
$$

In a domain $\Omega \subset \mathbb{R}^{n}$ it is better to define the fractional Laplacian in terms of the following bilinear form:

$$
\int_{\Omega}(-\Delta)^{s} u(\mathbf{x}) v(\mathbf{x}) d \mathbf{x}=\frac{c_{n, s}}{2} \underset{\left(\Omega \times \mathbb{R}^{n}\right) \cup\left(\mathbb{R}^{n} \times \Omega\right)}{\int} \frac{(u(\mathbf{x})-u(\mathbf{y}))(v(\mathbf{x})-v(\mathbf{y}))}{|\mathbf{x}-\mathbf{y}|^{n+2 s}} d \mathbf{y} d \mathbf{x}
$$

for $u, v$ belonging to the Sobolev space $H^{s}(\Omega)$. When $s=1$ this operator corresponds to the differential Laplacian operator $\Delta u=\partial_{x_{1}}^{2} u+\cdots+\partial_{x_{n}}^{2} u[46,47]$ with Neumann boundary conditions [14].

Let us now move on to the diffusive process on a discrete space. Let $G=(V, E)$ be a simple, connected graph with $|V|=N$ nodes and $|E|=m$ edges. The degree of a node in $V$ is the number of its nearest neighbors. Let dist $(v, w)$ be the shortest path distance between the nodes $v \in V$ and $w \in V$, i.e., the number of edges in one shortest path connecting both nodes. Let $d_{\max }=\max _{v, w}$ dist $(v, w)$ be the maximum distance between any pair of nodes in $G$, i.e., the graph diameter.

Hopping between the nodes in the discrete space of a network occurs through sinks (dark blue area in Figure 2) and sources (yellow area), where particles are allowed to hop to nonnearest nodes with a certain probability. Since the metaplexes that we study are undirected, the sinks also act as sources, and vice versa (see Supplementary Note 2). The evolution of the density of the diffusive particle among the nodes of the graph is controlled by the generalized diffusion equation [19],

$$
\frac{d \mathbf{u}(t)}{d t}=-\left(\sum_{d=1}^{d_{\max }} c_{d} \Delta_{d}\right) \mathbf{u}(t), \quad \mathbf{u}(0)=\mathbf{u}_{0},
$$

where $\Delta_{d}$ is the $d$-path Laplacian operator of the graph transformed by the coefficients $c_{d}$. This operator is defined as follows. Let $C(V)$ denote the set of complex-valued functions in $V$. Let $f \in C(V)$; then [19]

$$
\Delta_{d} f(v)=\sum_{w \in V, \operatorname{dist}(v, w)=d}(f(v)-f(w)), \quad v \in V .
$$

Notice that if we consider only dist $(v, w)=1$ in the above definition, this operator becomes the graph Laplacian $\Delta_{1}=L=K-A$, where $K$ is a diagonal matrix of node degrees and $A$ is the adjacency matrix of the graph. The transformation of the $\Delta_{d}$ operators through the coefficients $c_{d}$ allows one to tune the hopping of the diffusive particle between nearest and nonnearest neighbors in the graph. For instance, let

$$
\Delta_{M} f:=\sum_{d=1}^{d_{\max }} d^{-s} \Delta_{d} f
$$

designate the Mellin-transformed $d$-path Laplacians of the graph where $d_{\max }$ is the diameter of the graph.. Then, when $s \rightarrow \infty$, the transformed operator tends to $\Delta_{1}=$ $L=K-A$. However, when $s$ is relatively small the diffusive particle can hop not only 
to nearest neighbors but also to distant nodes with a certain probability that decays with the shortest path distance separating the origin and destination of the particle. It was recently proved that in the one-dimensional lattice when $s \in(1,3)$ there is a superdiffusive process on the graph [22]. The same happens for two-dimensional lattices when $s \in(2,4)[23]$. Other transforms, such as the exponential transform, also known as the Laplace transform:

$$
\Delta_{e} f:=\sum_{d=1}^{d_{\max }} e^{-d s} \Delta_{d} f
$$

can also be used in the generalized diffusion equation, but they have been proved to show no superdiffusive processes on the graph [22].

In the analysis of diffusion processes on metaplexes we will refer to the rate at which the diffusive particle reaches the steady state $\bar{u}$ as $t \rightarrow \infty$. The rate of convergence of the diffusion on the metaplex is controlled by the standard deviation of the density,

$$
\sigma(t)=\sqrt{\frac{1}{N-1} \sum_{i}\left(\int_{\Omega_{i}} u_{i}\left(t, \mathbf{x}_{i}\right)-\bar{u}_{i}\right)^{2}},
$$

where the summation is over all $N$ nodes in $V$. In our examples the steady state $\bar{u}$ is the uniform distribution on $V$.

3. Metaplexes: Structure and Dynamics. We defined informally a metaplex above. Here we present a formal definition of a metaplex and of a dynamical system on it. Let us start with the following definition.

Definition 3.1. A metaplex is a 4-tuple $\Upsilon=(V, E, \mathcal{I}, \omega)$, where $(V, E)$ is a graph, $\omega=\left\{\Omega_{j}\right\}_{j=1}^{k}$ is a set of locally compact metric spaces $\Omega_{j}$ with Borel measures $\mu_{j}$, and $\mathcal{I}: V \rightarrow \omega$.

For instance, let us consider a simple metaplex in which $(V, E)$ is a path graph, e.g., the path graph of three nodes with each node given by the unit disk $B(0,1) \subset \mathbb{R}^{2}$. In this case $\omega=\{B(0,1)\}$ and $\mathcal{I}$ is constant. The resulting metaplex is illustrated in Figure 2. The endostructure of this metaplex is given by the internal structure inside the unit disk, and its exostructure is given by the connectivity of the three nodes in the form of a path. In the current work we focus only on metaplexes in which the internal structure of the nodes is continuous; that is, when $\omega$ is a set of open domains $\Omega_{j} \subset \mathbb{R}^{n}$, each endowed with the Lebesgue measure. Other scenarios in which the internal structure is a discrete space, not just a graph, should be considered in a separate analysis and will not be treated in the current work. Now we can define a dynamical system on a metaplex.

Definition 3.2. A dynamical system on a metaplex $\Upsilon=\left(V, E, \mathcal{I}, \omega=\left\{\Omega_{k}\right\}\right)$ is a tuple $(\mathcal{H}, \mathcal{T})$. Here $\mathcal{H}=\left\{H_{v}: L^{2}\left(\Omega_{\mathcal{I}(v)}, \mu_{\mathcal{I}(v)}\right) \rightarrow L^{2}\left(\Omega_{\mathcal{I}(v)}, \mu_{\mathcal{I}(v)}\right)\right\}_{v \in V}$ is a family of operators such that the initial value problem $\partial_{t} u_{v}=H_{v}\left(u_{v}\right),\left.u_{v}\right|_{t=0}=$ $u_{0}$, is well-posed, and $\mathcal{T}=\left\{T_{v w}\right\}_{(v, w) \in E}$ is a family of bounded operators $T_{v w}$ : $L^{2}\left(\Omega_{\mathcal{I}(v)}, \mu_{\mathcal{I}(v)}\right) \rightarrow L^{2}\left(\Omega_{\mathcal{I}(w)}, \mu_{\mathcal{I}(w)}\right)$.

There are many possible dynamical systems that can be considered on a metaplex under the previous general definition. In the current work we focus only on the study of diffusive processes at the endo- and exostructures of the metaplexes. In this scenario we consider a continuous diffusion equation inside the nodes where the evolution of 
the density of the diffusive particle is controlled by the fractional Laplacian operator as described in section 2. At the exoskeleton we consider a diffusive process in which the particle hops from one node to another controlled by the $d$-path Laplacian of the graph also described in section 2. The in-and-out motion of the diffusive particle between nodes is carried out through the so-called sources and sinks inside the nodes. A source is a subdomain inside the node from which a diffusive particle can emerge to the interior of the node. A sink is another (not necessarily different) subdomain inside the node from which a diffusive particle can abandon the interior of the node toward another node in the metaplex.

In the simplest case, diffusive particles move between different nodes through such sinks and sources in the interior, corresponding to a coupled system of diffusion equations for the density $u_{j}(t, \mathbf{x})$ of particles in the node $v_{j} \in V$ :

$$
\begin{aligned}
\partial_{t} u_{j}(t, \mathbf{x})=\operatorname{div} & J_{j}\left(u_{j}(t, \mathbf{x})\right)-\sum_{i:\left(v_{j}, v_{i}\right) \in E} \alpha_{i j}(\mathbf{x}) u_{j}(t, \mathbf{x}) \\
& +\sum_{i:\left(v_{i}, v_{j}\right) \in E} \alpha_{j i}\left(\psi_{j i}^{-1}(\mathbf{x})\right) \operatorname{det}\left(\nabla \psi_{j i}^{-1}\right) u_{i}\left(t, \psi_{j i}^{-1}(\mathbf{x})\right)
\end{aligned}
$$

for $(t, \mathbf{x}) \in(0, \infty) \times \Omega_{j}$. Here $J_{j}$ is the flux of particles and $\operatorname{div} J_{j}$ is the generator of the diffusion process in $\Omega_{j}$, for example, $\operatorname{div} J_{j}=\Delta$ in the case of a normal diffusion, while $\operatorname{div} J_{j}=-(-\Delta)^{s}$ for the superdiffusive Lévy process. The edges $\left(v_{i}, v_{j}\right) \in E$ are realized by a map $\psi_{j i}: \Omega_{j} \rightarrow \Omega_{i}$ that specifies the jumps between domains, and the coefficients $\alpha_{j i}(\mathbf{x})$ are transition rates from $\Omega_{i}$ to $\Omega_{j}$ : Particles jump from $\mathbf{x}$ to $\psi_{j i}(\mathbf{x})$ with amplitude $\alpha_{i j}(\mathbf{x})$ and from $\psi_{j i}(\mathbf{x})$ to $\mathbf{x}$ with amplitude $\alpha_{j i}\left(\psi_{j i}^{-1}(\mathbf{x})\right)$. Physically, the system (3.1) arises for nodes that correspond to spatially distant domains.

For the system of diffusion equations $(3.1), H_{v_{j}} u_{j}=\operatorname{div} J_{j}\left(u_{j}\right)$, while the $T_{v w}$ correspond to the entries of the transition matrix of the network given by the functions $\alpha_{i j}$.

Diffusion in a network may be studied from its generator, the graph Laplacian $\Delta_{1}$. A dynamical system in a metaplex is similarly described by an operator matrix $\mathcal{D}$. For diffusion processes like (3.1) it takes the abstract form

$$
\partial_{t} u=\mathcal{D} u \text {. }
$$

$\mathcal{D}$ is an $N \times N$ block operator matrix of unbounded operators on the product space $\bigoplus_{j=1}^{N} L^{2}\left(\Omega_{j}, \mu_{j}\right)=L^{2}\left(\Omega_{1}, \mu_{1}\right) \times \cdots \times L^{2}\left(\Omega_{N}, \mu_{N}\right)$, with $N$ the number of nodes in $V$. In line with Definition 3.2 we write $\mathcal{D}=H+T$, where

$$
H=\left(\begin{array}{ccccc}
\operatorname{div} J_{1} & 0 & 0 & \cdots & 0 \\
0 & \operatorname{div} J_{2} & 0 & \cdots & 0 \\
\vdots & 0 & \ddots & & \vdots \\
\vdots & \vdots & & \ddots & \vdots \\
0 & 0 & \cdots & \cdots & \operatorname{div} J_{N}
\end{array}\right)
$$

is given by the operators $H_{v_{j}} u_{j}=\operatorname{div} J_{j}\left(u_{j}\right)$ and

$$
T=\left(\begin{array}{cccc}
\alpha_{11} T_{11} & \alpha_{12} T_{12} & \cdots & \alpha_{1 N} T_{1 N} \\
\alpha_{21} T_{21} & \alpha_{22} T_{22} & \cdots & \alpha_{2 N} T_{2 N} \\
\vdots & \vdots & \ddots & \vdots \\
\alpha_{N 1} T_{N 1} & \alpha_{N 2} T_{N 2} & \cdots & \alpha_{N N} T_{N N}
\end{array}\right)
$$

Copyright (c) by SIAM. Unauthorized reproduction of this article is prohibited. 
describes the network diffusion defined by $\mathcal{T}$. Provided the adjoint

$$
T^{*}\left(\begin{array}{c}
1 \\
1 \\
\vdots \\
1
\end{array}\right)=0,
$$

particles are conserved. Here the $T_{i j}$ are transition operators between $\Omega_{i}$ and $\Omega_{j}$, as given by the sources and sinks, and $\alpha_{i j}$ are the transition probabilities.

For a network, spectral properties of the network Laplacian determine the longtime behavior of diffusion. Analogously, the long-time behavior of the linear diffusion equation (3.2) in the metaplex is determined by the spectral properties of $\mathcal{D}$ [62]. While the spectrum of $H$ is determined by the internal structure of the nodes, the spectrum of $T$ combines the details of the location and strength of sinks, respectively, sources, with the external network structure.

In addition to the coupling of sinks and sources as in (3.1), numerous other types of couplings can be considered within the framework of metaplexes. While their detailed analysis is beyond the scope of this paper, we mention two important examples.

The first example is a special case of (3.1), with all nodes of the same geometry $\Omega=\Omega_{j}$ for every $j$ and local transitions $\psi_{j i}=\mathrm{Id}$, where Id is the identity operator (matrix) of the appropriate dimension. In this case the density $u$ may be interpreted as a vector-valued function $u=\left(u_{1}, \ldots, u_{N}\right):[0, \infty) \times \Omega \rightarrow \mathbb{R}^{N}$, and the network encodes the dynamics of the "internal state" of the particle described by a vector in $\mathbb{R}^{N}$. In biology, such processes are of interest in describing the diffusion of complex organisms [54, 18, 17].

In complex systems such as road systems or the transport of chemicals between cells, the coupling between the nodes occurs through the boundary $\partial \Omega_{j}$, not through internal sinks and sources. Every edge $\left(v_{i}, v_{j}\right) \in E$ is physically realized by open entrances and exits $\Gamma_{i j} \subset \partial \Omega_{i}$ and $\Gamma_{j i} \subset \partial \Omega_{j}$, together with a homeomorphism $\phi_{i j}: \Gamma_{i j} \rightarrow \Gamma_{j i}$ identifying points between them. We define $\Gamma_{i 0}=\partial \Omega \backslash \bigcup_{j} \Gamma_{i j}$. If there is an edge between $\Omega_{i}$ and $\Omega_{j}$, particles leave $\Omega_{i}$ through $\Gamma_{i j}$ and arrive at $\Gamma_{j i}$ in $\Omega_{j}$.

The resulting system of diffusion equations is coupled through the boundary conditions with a Kirchhoff's law: For $\mathrm{x} \in \bigcup_{i} \Gamma_{j i}$,

$$
\begin{gathered}
u_{j}(t, \mathbf{x})=\sum_{i: \mathbf{x} \in \Gamma_{j i}} \alpha_{j i} \operatorname{det}\left(\nabla_{\partial \Omega} \phi_{j i}\right) u_{i}\left(t, \phi_{j i}(\mathbf{x})\right), \\
J\left(u_{j}(t, \mathbf{x})\right) \cdot \nu_{j}(\mathbf{x})=-\sum_{i: x \in \Gamma_{j i}} \alpha_{j i} \operatorname{det}\left(\nabla_{\partial \Omega} \phi_{j i}\right) J\left(u_{i}\left(t, \phi_{j i}(\mathbf{x})\right)\right) \cdot \nu_{i}\left(\phi_{j i}(\mathbf{x})\right),
\end{gathered}
$$

where $\nu_{j}$ is the exterior unit normal vector. The number of particles is preserved if the transition probabilities satisfy $\sum_{i} \alpha_{j i}=1$ for every $j$.

\section{Operators and Spectra for Metaplexes.}

4.I. Variation of Eigenvalues with Fractional Exponent and Spatial Scale. Note that $H=\bigoplus_{j=1}^{N} H_{j}=\bigoplus_{j=1}^{N} \operatorname{div} J_{j}$ is a block diagonal operator matrix acting on $L^{2}\left(\Omega_{1}\right) \times \cdots \times L^{2}\left(\Omega_{N}\right)$. A basis of eigenfunctions of $H$ is constructed from bases $\left\{u_{j, k}\right\}_{k}$ of eigenfunctions of $\operatorname{div} J_{j}$ in $\Omega_{j}$ and is given by $\left\{u_{j, k} e_{j}\right\}_{j, k}$, where the subscripts denote the $k$ th eigenfunction in the domain $\Omega_{j}$. Here, $e_{j}$ denotes the $j$ th standard unit vector in $\mathbb{R}^{N}$. 
If $H_{j}=-(-\Delta)^{s_{n o d}}$ is the fractional Laplacian in $\Omega_{j}$ with Neumann boundary conditions, the eigenvalues of $H_{j}$ are homogeneous functions of the spatial scale, i.e., if the spatial variable is scaled, then so also is the function, $f(\alpha x)=\alpha^{p} x$, where $p$ is the degree of homogeneity. More precisely, the bilinear form of $H_{j}$ is homogeneous under scaling $\Omega_{j} \mapsto \Lambda \Omega_{j}, \Lambda>0$. For instance, if $\Omega_{j}=B(0,5)$ is a ball of radius 5 , then the scaling of the domain can be interpreted as $\Lambda \Omega_{j}=B(0, \Lambda 5)$.

From their characterization in terms of a Rayleigh quotient, the eigenvalues in $\Lambda \Omega_{j}$ are given by $\left\{\Lambda^{-2 s_{\text {nod }}} \lambda_{j, k}\right\}_{k=1}^{\infty}$, if $\left\{\lambda_{j, k}\right\}_{k=1}^{\infty}$ are the eigenvalues of $H_{j}$ in $\Omega$ [13]. Only the lowest eigenvalue $\lambda_{j, 1}=0$ is fixed under this scaling.

We see that for small domains, $\Lambda \rightarrow 0$, the spectral gap $\lambda_{j, 2}-\lambda_{j, 1}$ in the node $\Omega_{j}$ increases with $s_{n o d}$, and therefore Brownian motion in the nodes gives the fastest convergence to equilibrium. For large domains, $\Lambda \rightarrow \infty$, the spectral gap $\lambda_{j, 2}-\lambda_{j, 1}$ in the nodes $\Omega_{j}$ decreases with $s_{n o d}$, and therefore the long jumps of the fractional diffusion lead to equilibration faster than Brownian motion inside each node.

In a later section (see section 6) we will illustrate these findings computationally.

4.2. Weakly and Strongly Interacting Limits. The spectral properties of $\mathcal{D}_{\varepsilon}=$ $H+\varepsilon T$ are most easily understood in the limit $\varepsilon \rightarrow 0$ of weak network interactions between the nodes, and in the strong network interactions for $\varepsilon \rightarrow \infty$. In the former case, a particle is trapped for a long time inside the domain in which it was initially placed. The time scale of the slow global equilibration is determined by $T$. In the latter case, the transient dynamics is governed by the dynamics of the network and the geometry of the sinks and sources, i.e., $T$, but the dynamics $H$ inside the nodes determines the long-time approach to equilibrium.

From standard perturbation theory, for general, symmetric interactions $T$ the stationary states corresponding to $\lambda_{j, 1}=0, j \in\{1, \ldots, N\}$, split into eigenvalues $\varepsilon \widetilde{\lambda}_{k}+o(\varepsilon)$ according to the eigenvalues $\tilde{\lambda}$ of $T$ restricted to the kernel of $H$. The kernel of $H$ is spanned by the constant functions 1 in $\Omega_{j}, j=1, \ldots, N$. Hence, $\left(\left.T\right|_{\text {ker } H}\right)_{i j}=\frac{1}{\sqrt{\left|\Omega_{i}\right|\left|\Omega_{j}\right|}} \int_{\Omega_{j}} \alpha_{i j} T_{i j} 1 d x, i, j=1, \ldots, N$, is given by an effective graph Laplacian for the metaplex, in which the internal structure has been integrated out. The eigenvalues $\widetilde{\lambda}_{k}$ of this matrix determine the spectral gap $\varepsilon\left(\widetilde{\lambda}_{2}-\widetilde{\lambda}_{1}\right)$ in terms of the spectral gap of the effective graph Laplacian $\left.T\right|_{\text {ker } H}$, independent of $H$. Higher eigenvalues will depend on the location of the sources and sinks and the eigenfunctions of $H_{j}$.

Multiple eigenvalues arise, in particular, when several of the $H_{j}$ coincide. In this case the eigenvalues are determined by the restriction $T_{r}$ of $T$ to the subnetwork of those nodes where the internal diffusion has the eigenvalue $\lambda_{j, k}$.

Higher eigenvalues depend on the location of the sources and sinks. The spectral gap of the network is $\varepsilon \lambda_{2}$ and thus independent of the diffusion process $H$.

Local equilibration within the nodes, however, happens on faster time scales, with little effect of the coupling. For small $\varepsilon$, the gap between the higher bands $\lambda_{j, k}+\varepsilon \lambda_{n}^{r}$ and the equilibrium is determined by the diffusion $H$, up to terms of order $\varepsilon$, where $\left\{\lambda_{k}^{r}\right\}$ are the eigenvalues of $T_{r}$.

The case where $T_{i j}=$ Id is discussed in the Supplementary Material (Note 3). For general $T_{i j}$ the spectrum of the network interaction operator $T$ will be considered elsewhere.

4.3. Numerical Results of the Spectral Properties. We illustrate the spectral gap of $\mathcal{D}_{\varepsilon}$ for a linear network of 11 unit disks $\Omega=\Omega_{j}=B(0,1) \subset \mathbb{R}^{2}$. The lowest 10 nonzero eigenvalues are considered as a function of $\varepsilon$, the coupling operator $T$, 


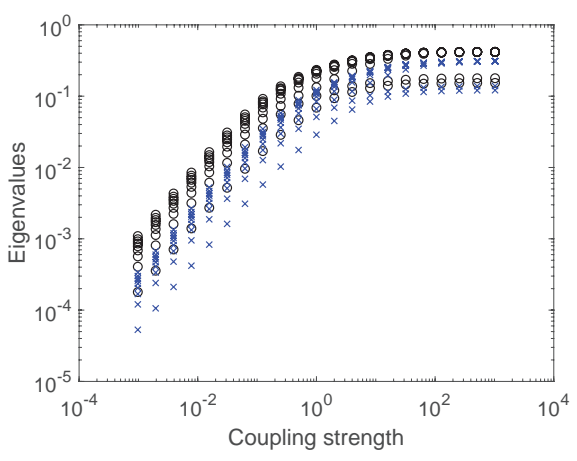

Fig. 3 Comparison of eigenvalues for the case of different discretizations $h$ of the mesh: a coarse mesh (347 degrees of freedom) (o) and a finer mesh (1325 degrees of freedom) $(\times)$. Here $s_{\text {net }}=0.8$ and $s_{\text {nod }}=0.4$ and we consider different coupling points.

and the diffusion $H$. We choose the generator $H_{j}$ of the diffusion process inside $\Omega_{j}$ as a fractional Laplacian $-(-\Delta)^{s_{n o d}}$ with Lévy exponent $s_{\text {nod }}$ and approximate it by finite elements on quasi-uniform spatial meshes. See Figure 6 for a plot of the mesh and [24] for the approximation of the fractional Laplacian.

The network coupling $T_{i j}$ is taken to be a $d$-path Laplacian (2.7) with hopping between $\Omega_{i}$ and $\Omega_{j}$ proportional to an exponential $2^{-s_{n e t}|i-j|}$.

As $H$ is unbounded, the eigenvalues of its discretization extend over several orders of magnitude, while the spectral gap is tiny. To resolve this, the discretization of the mesh $h$ needs to be small compared to the strength of the coupling, and standard MATLAB routines do not identify the bounded branches of eigenvalues obtained in subsection 4.2 for large $\varepsilon$. Discretization errors significantly increase as $s_{\text {nod }} \rightarrow 0$ because the smoothness of the solutions in $\Omega$ decreases.

Figure 3 indicates the complications inherent in approximating the spectrum of a large system of differential operators. The lowest ten nonzero eigenvalues of $\mathcal{D}_{\varepsilon}$ are depicted for the exponential coupling as a function of the coupling strength $\varepsilon \in$ $\left[2^{-10}, 2^{10}\right]$ for meshes of 347 and 1325 degrees of freedom, $s_{\text {net }}=0.8, s_{\text {nod }}=0.4$. We consider a prototypical metaplex coupling as depicted in Figure 2 for $\alpha_{i j}=10$. While results agree for large coupling strengths, the spectral gap is significantly smaller for the finer mesh at small coupling. Nevertheless, the qualitative behavior from subsection 4.2 is recovered: the gap increases linearly for small $\varepsilon$, and for large $\varepsilon$ it converges to the lowest eigenvalue of $H$.

Figure 4 connects the theory of subsection 4.2 with the numerical results from section 6 and shows the effect of the geometry of the nodes on the dynamics of the network. In the case of small nodes (Figure 4, left) when the coupling is weak, the dynamics of the network is dominated by the diffusion process inside the nodes and we have almost no hopping between domains. As we increase the coupling strength, for $s_{\text {nod }}=0.8$ the spectral gap is bigger than that for $s_{\text {nod }}=0.2$, in agreement with subsection 4.1. Similar to the case of multiplex networks, the first nonzero eigenvalue of $\mathcal{D}_{\varepsilon}$ is related to the equilibration time in the whole network, i.e., $t=\lambda_{2}^{-1}$ [61]. From Figure 4 (left), the equilibration time for the case $s_{\text {nod }}=0.2$ is much longer than for the normal diffusion case inside the nodes. In section 6 we obtain computational results that confirm these findings. 

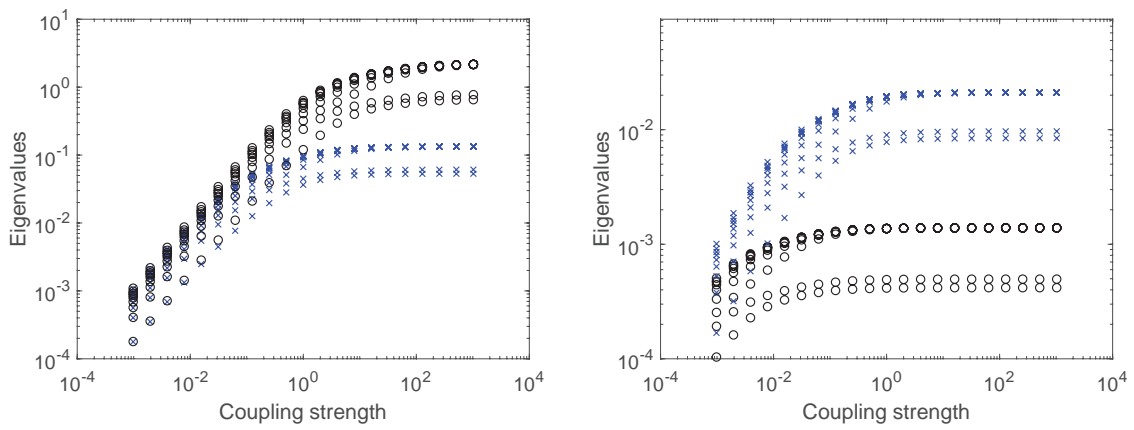

Fig. 4 Eigenvalues for small (left) and big (right) nodes. In both cases, $s_{\text {net }}=0.8$ with $s_{\text {nod }}=0.2$ (crosses) and $s_{\text {nod }}=0.8$ (circles).

In the case of big nodes (Figure 4, right), the spectral gap decreases as we increase the parameter $s_{\text {nod }}$. This means that, contrary to the small node case, a metaplex with superdiffusion inside the nodes $\left(s_{n o d}=0.2\right)$ reaches equilibrium faster than for internal Brownian motion.

5. Analysis of Diffusion in Metaplexes. In this section we show how PDE techniques allow for the analysis of the time-dependent diffusion introduced in section 3 in a metaplex of bounded domains $\omega=\left\{\Omega_{j} \subset \mathbb{R}^{n}\right\}_{j=1}^{k}$, as illustrated in Figure 2 . We compare the interaction of diffusion, respectively, superdiffusion, in the nodes with either short or long range coupling in the external network. While for general complex networks the connectivity and large scale geometry of the network will crucially influence the dynamics, here we focus on simple linear metaplexes $V=\mathcal{Q}=\{\ldots,-2,-1,0,1,2, \ldots\}$ and short times.

More precisely, we assume that the diffusion process in each node $\Omega_{j}$ is governed by either a fractional Laplacian $H_{j}=-(-\Delta)^{s_{\text {nod }}}$ or a self-adjoint elliptic differential operator of second order like the Laplacian, $H_{j} u_{j}=\operatorname{div}\left(a \nabla u_{j}\right), a \in C^{\infty}\left(\bar{\Omega}_{j}\right)$, with Neumann boundary conditions. The network coupling $T_{i j}$ consists of disjoint sinks and sources, as in Figure 2. The amplitude is chosen according to the $d$-path Laplacian (2.6)-(2.7), with hopping between $\Omega_{v}$ and $\Omega_{w}$ proportional to $\operatorname{dist}(v, w)^{-s_{n e t}}$ (long range coupling), respectively, $2^{-s_{\text {net }} \mathrm{dist}(v, w)}$ (short range coupling).

When we consider a metaplex we are dealing with the endodynamics occurring inside the nodes and the exodynamics occurring between them. Inside each node $\Omega_{j}$ the solution $e^{t H_{j}} u_{j, 0}$ to the diffusion equation for $H_{j}$ with initial condition $u(t=0)=$ $u_{j, 0}$ is an integral operator $\left(e^{t H_{j}} u_{0}\right)(t, \mathbf{x})=\int_{\Omega} K_{H_{j}}(t, \mathbf{x}, \mathbf{y}) u_{0}(\mathbf{y}) d \mathbf{y}$. The integral kernel $K_{H_{j}}$ describes the evolution of a Dirac point mass in $\mathbf{y}$ at time $t=0$. For ordinary diffusion away from the boundary $\partial \Omega_{j}$ it satisfies the Gaussian estimate

$$
\left|K_{H_{j}}(t, \mathbf{x}, \mathbf{y})\right| \leq C t^{-n / 2} e^{-C \frac{|\mathbf{x}-\mathbf{y}|^{2}}{t}}
$$

for some $C>0$, analogous to the explicit solution formula for the diffusion equation on $\mathbb{R}^{n}$ [53]. Similar bounds are known for ordinary diffusion on metric measure spaces $\Omega_{j}$ [32]. Fractional diffusion, on the other hand, exhibits slow algebraic decay in the form of a Poisson estimate

$$
\left|K_{H_{j}}(t, \mathbf{x}, \mathbf{y})\right| \leq C t\left(|\mathbf{x}-\mathbf{y}|+t^{1 /\left(2 s_{n o d}\right)}\right)^{-n-2 s_{n o d}}
$$


away from $\partial \Omega_{j}[28]$. In a convex domain the estimate is sharp for short times, and it allows us to estimate the diffusion between nodes across the network.

Consider the linear network in Figure 2, with an initially uniform density localized in $\Omega_{1}$. From (3.1) we calculate the change of the total density in node $j$ :

$\left.\partial_{t}\right|_{t=0} \int_{\Omega_{j}} u_{j}(t, \mathbf{x}) d \mathbf{x}=-\frac{\delta_{j 1}}{\left|\Omega_{1}\right|} \sum_{i} \int_{\Omega_{j}} \alpha_{i j}(\mathbf{x}) d \mathbf{x}+\frac{1}{\left|\Omega_{1}\right|} \int_{\Omega_{j}} \alpha_{j 1}\left(\psi_{j 1}^{-1}(\mathbf{x}) \operatorname{det}\left(\nabla \psi_{j 1}^{-1}\right) d \mathbf{x}\right.$.

Thus, initially particles hop from node 1 to node $j$ according to the transition probabilities $\alpha_{i j}$ of the network, from $\mathbf{x}$ to $\psi_{j 1}(\mathbf{x})$. From Duhamel's formula for the solution of the inhomogeneous diffusion equation, they then evolve inside $\Omega_{j}$ according to $K_{H_{j}}$ before jumping back to node 1 , or further to a different node $k$. Both processes only enter into the following quadratic term of the Taylor expansion in $t$. These formal arguments are made rigorous in terms of an asymptotic expansion for $t \rightarrow 0$ of the heat kernel $K_{\mathcal{D}}$ for the network of interacting domains; see, e.g., [45].

Compared to nodes without internal structure, hopping to node $k$ is reduced by the rate $K_{H_{j}}\left(\tau, \psi_{j 1}(\mathbf{x}), \mathbf{y}\right)$ of diffusing from $\psi_{j 1}(\mathbf{x})$ to a point $\mathbf{y}$ in the region of the sink to node $k$ within time $\tau$. The internal diffusion thus always slows down the network dynamics if sources and sinks are disjoint.

The exit time $\tau_{\text {source, sink }}^{j}$ taken to get from source to sink is well studied for both normal and fractional diffusion [2]. It satisfies a (fractional) Fokker-Planck equation and satisfies the estimate (5.1), respectively, (5.2), for short times. For long times, $\tau_{\text {source, sink }}^{j}$ decays exponentially fast in time.

For short times, the dependence on the internal diffusion $H_{j}$, the geometry of $\Omega_{j}$, and the location of sinks and sources are well described by (5.1) and (5.2). For sources and sinks that are far apart, in the case of normal diffusion (5.1) shows a faster than exponential suppression of the transition rate due to the internal structure. Fractional diffusion suppresses the transition rate algebraically in the distance between sink and source, according to (5.2). The smaller $s_{n o d}$ is, the faster the equilibration and the smaller the suppression in $\Omega_{j}$.

Similarly, if $T_{i j}$ is nonzero only for $|i-j| \leq 1$, hopping to the next $j$ th nearest neighbors is suppressed $j$ times by the internal diffusion. Starting from an initial density localized in node 1 , the total density in node $j$ will be exponentially small: $\int_{\Omega_{j}} u_{j}(t, \mathbf{x}) d \mathbf{x} \leq C e^{-C|j-1|}$ for some $C=C(t)>0$ depending on the endostructure.

6. Numerical Analysis of Diffusion in Metaplexes. This section investigates the influence of the internal structure of the nodes on the global metaplex dynamics using numerical experiments for the toy example of a linear metaplex. For the localized coupling $T_{i j}$ in a given area (Figure 5) we show the rich dependence on the internal structure in a metaplex, such as the geometry of the nodes and the nature of the coupling, and we place the results, which were obtained in certain limits, into a wider perspective. For example, a superdiffusive process in the nodes may slow down equilibration in the metaplex; see Experiment 1. On the other hand, the network diffusion dominates the qualitative global behavior: In subsection 6.3.1 we show that network superdiffusion due to long range hopping survives independently of the internal structure. This section illustrates the rich phenomena that arise from the interplay of the exo- and endostructures of the metaplex. See the supplementary material for numerical and analytical results for the case $T_{i j}=\mathrm{Id}$. The conclusions are not restricted to the linear metaplex, but will be studied in what follows for some real-world examples. 


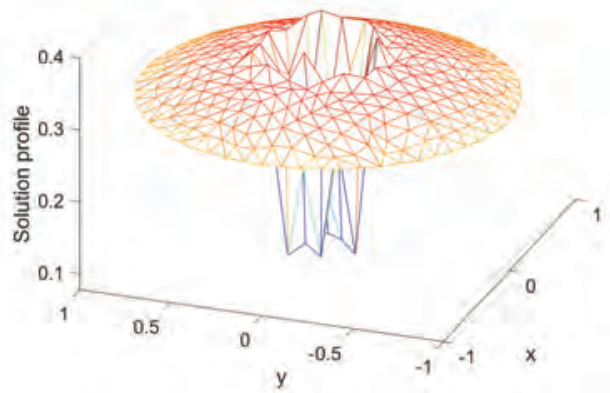

(a)

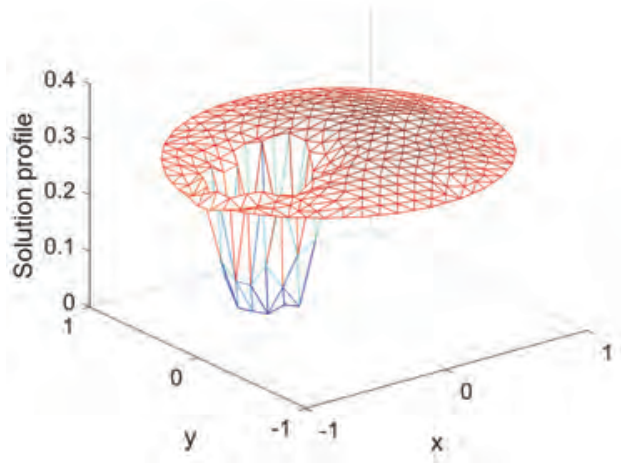

(b)

Fig. 5 Density profile for different coupling points between nodes.

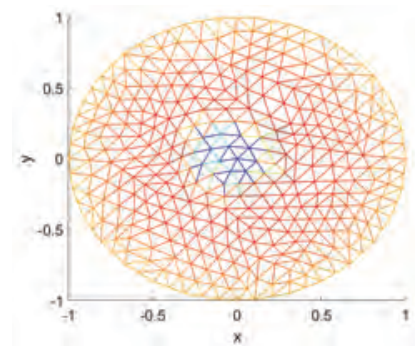

(a)

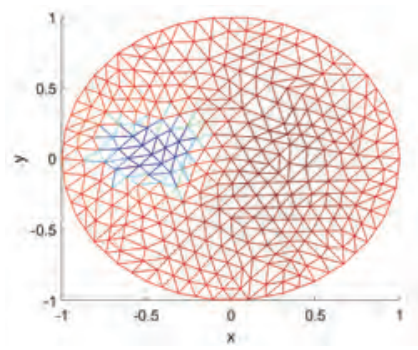

(b)

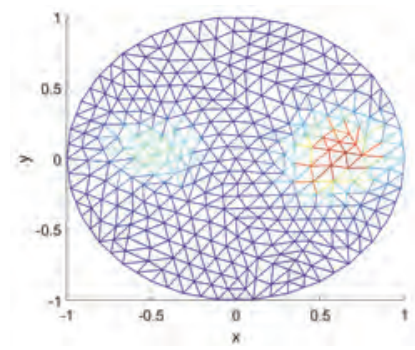

(c)

Fig. 6 Mesh inside the nodes for the different coupling areas in Experiment 1(a) and Experiment $2(\mathrm{~b}, \mathrm{c})$.

6. I. Setup of Numerical Experiments. Here we analyze a simple metaplex consisting of 51 identical circular domains $\Omega=\Omega_{j} \subset \mathbb{R}^{2}$ connected in the form of a linear chain, i.e., a path graph. The nodes are labeled in consecutive order starting with one. Starting from a uniform distribution in node 1, we study the evolution of the density in the nodes depending on the network and the internal diffusion processes, the size of the nodes, and the strength and nature of the coupling between the nodes.

Two different sizes of nodes are considered, $\Omega_{s}=B(0,1)$ and $\Omega_{b}=B(0,100)$. These different sizes will also be used when we move to the analysis of real-world metaplexes. The diffusion equation (3.1) inside each node with Lévy exponent $s_{\text {nod }}$ is approximated by finite elements in space on a quasi-uniform spatial mesh with 347 degrees of freedom. See Figure 6 for a plot of the mesh and [24] for the numerical approximation of the fractional Laplacian. For the time discretization we use a backward Euler method in time with a sufficiently small, fixed time step $d t=0.01$.

Unless stated otherwise, the network coupling between nodes $(i, j)$ is taken to be of short range, according to the Laplace-transformed $d$-path Laplacian (2.7). The coupling strength between nodes $(i, j)$ is thus proportional to $2^{-s_{n e t}|i-j|}$ for different exponents $s_{\text {net }}$. 


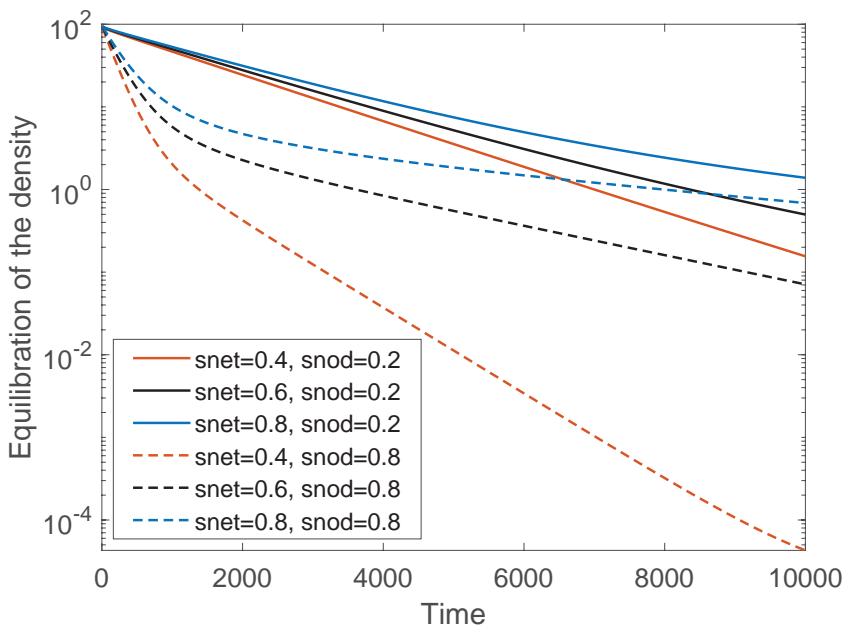

Fig. 7 Density equilibration in node 1 for central coupling region.

6.2. Experiment I: Central Coupling Region. For different values of the network exponent $s_{n e t}=0.4,0.8$, we compare close to normal diffusion (Lévy exponent $\left.s_{\text {nod }}=0.8\right)$ to superdiffusion $\left(s_{\text {nod }}=0.2\right)$ inside a metaplex of small nodes $\Omega_{s}$. In this experiment we consider that the nodes of the metaplex are connected by means of a central region of the node, as depicted in Figure 5(a), which simultaneously as a sink and as a source. See the supplementary material (Note 2) for a more detailed description of sinks and sources. The coupling strength is fixed to be $\alpha=10$.

Figure 7 shows the time at which the density in node 1 reaches the equilibrium given by $\left|\int_{\Omega_{1}} u_{1}(t, \mathbf{x}) d \mathbf{x}-\frac{1}{N} \int_{\Omega_{1}} u_{0}(\mathbf{x}) d \mathbf{x}\right|$, where $u(t, \mathbf{x})$ is the density in the node at a given time $t$ and $u_{0}(\mathbf{x})$ is the initial density in the metaplex. The figure illustrates the strong effect of the dynamics inside the nodes on the diffusion process, even though the network dynamics dominates. We see that superdiffusion inside each node slows down the diffusion in the network: Due to the nature of the Lévy process, particles quickly diffuse far away from the sink. As they take time to return and hop to the next node, diffusion between nodes in the whole network is slowed down. In subsection 6.3 we discuss how the size of the nodes affects this behavior.

The spatial localization of the sinks and sources decreases the total strength of the coupling, resulting in a slower equilibration of the densities in the node compared to the uniform coupling discussed in the supplementary material (Note 3). Changing the strength of a sufficiently strong localized coupling does not significantly affect this behavior, since the equilibration time saturates: independent of the coupling strength, particles that are located away from the sink cannot hop to a neighboring node.

In Figure 8 we show the evolution in time of the total density $\int_{\Omega_{j}} u_{j}(t, \mathbf{x}) d \mathbf{x}$ in each node $\Omega_{j}$. While superdiffusion inside the nodes has only limited effect on the equilibration of the densities in the network, it speeds up hopping to distant nodes. From Figure 8 we observe that when the network dynamics is dominated by superdiffusion $\left(s_{\text {net }}=0.4\right)$, only for short times does the superdiffusion inside the nodes (red crosses) overtake normal diffusion, while for longer times the equilibrium is attained faster for $s_{\text {nod }}=0.8$. 

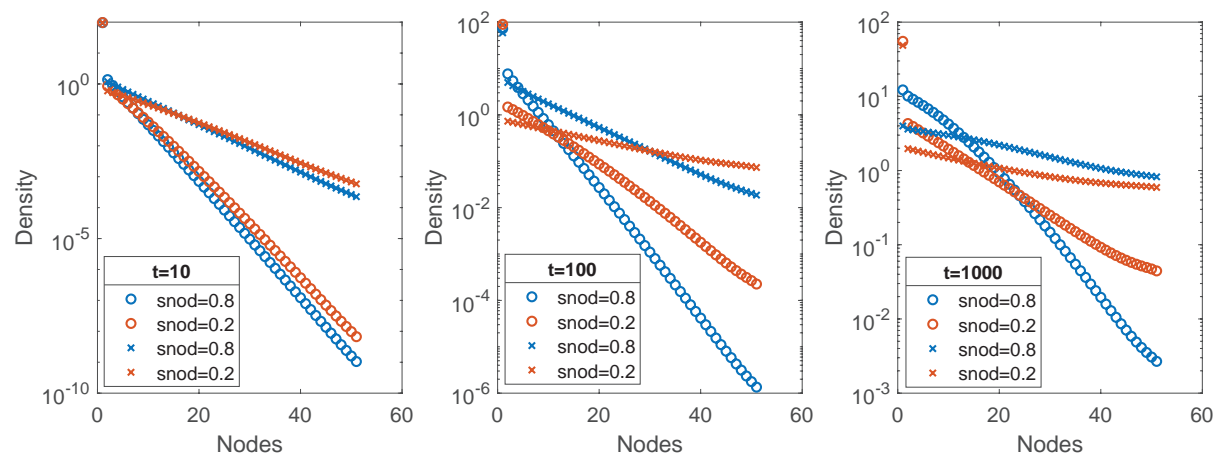

Fig. 8 Density distribution for central coupling region, $s_{n e t}=0.8(\circ)$, resp., $0.4(\times)$.

6.3. Experiment 2: Distant Coupling Regions. To study the effect of the geometry of the coupling and the node on the global dynamical process, we replace the central coupling from subsection 6.2 with a prototypical metaplex coupling as depicted in Figure 2: For the odd nodes the coupling region is located as in Figure 5(b), while for even nodes the coupling region is on the opposite side. Similar to the central coupling, the coupling regions act as sinks and as sources at the same time. The coupling areas in both domains are equal; therefore, in the case of $\Omega_{b}$, the areas are more localized and distant.

For the network dynamics, we consider the short range coupling from Experiment 1 given by the Laplace-transformed $d$-path Laplacian (2.7) with coupling between nodes $(i, j)$ proportional to $2^{-s_{n e t}|i-j|}, s_{\text {net }}=0.4,0.8$. The coupling strength is fixed to be $\alpha=10$ for $\Omega_{s}$ and $\alpha=100$ for $\Omega_{b}$. In subsection 6.3.1 we compare the resulting dynamics to a long range coupling proportional to $|i-j|^{-s_{n e t}}$, with the Mellin-transformed $d$-path Laplacian (2.6).

In this experiment, the distance between the sinks and sources also leads to a delay; particles appear in the node and have to diffuse to find a sink to hop to another node. Coupled with the exodynamics, the delay leads to small density oscillations between neighboring nodes. For clearer illustration, in Figures 10 to 13 we only consider the odd nodes of the metaplex.

Figure 9(a) shows the deviation of the density in node 1 from equilibrium, corresponding to Figure 7 in Experiment 1. Similar to Experiment 1, superdiffusion inside the small nodes $\Omega_{s}$ slows down equilibration. However, the evolution in time of the total density $\int_{\Omega_{j}} u_{j}(t, \mathbf{x}) d \mathbf{x}$ in nodes $\Omega_{i}$ is depicted in Figure 10 at times $t=10,100,1000$. One observes that superdiffusion in the nodes allows particles to reach distant nodes more efficiently than approximately normal diffusion $s_{\text {nod }}=0.8$. This confirms the interpretation in section 4 . The case $s_{n e t}=0.4$ in Figure 10 exhibits similar dynamics as in Experiment 1, where $s_{n o d}=0.8$ equilibrates the metaplex's density faster on long time scales.

We conclude that particles undergoing approximately normal diffusion $\left(s_{\text {nod }}=\right.$ $0.8)$ are slower, but more precise. Superdiffusing particles $\left(s_{\text {nod }}=0.2\right)$ explore the metaplex faster, but take more time to equilibrate the density across the whole metaplex. Similar observations have been made in [44], where the targeting efficiency of E. coli bacteria was studied in simulations. They observed that bacteria with higher motility, following a superdiffusion process, found targets faster, but were also at 


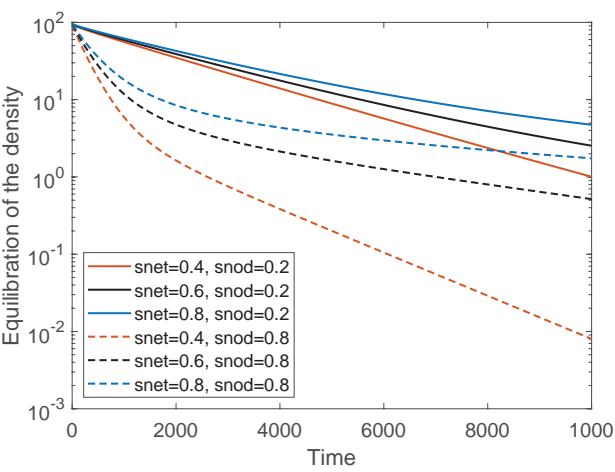

(a)

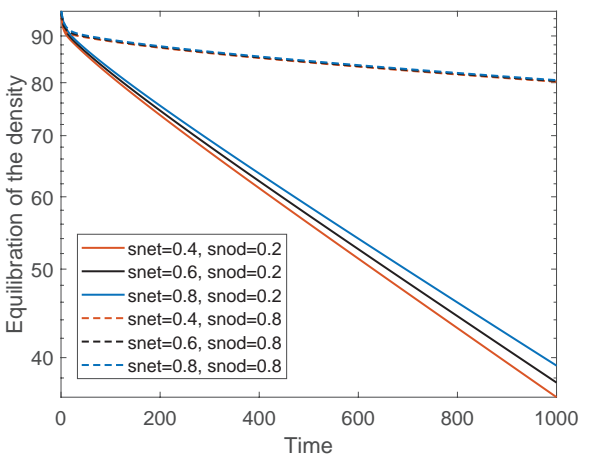

(b)

Fig. 9 Density equilibration in node 1 for disjoint sinks and sources (a) $\Omega_{s}$, (b) $\Omega_{b}$.
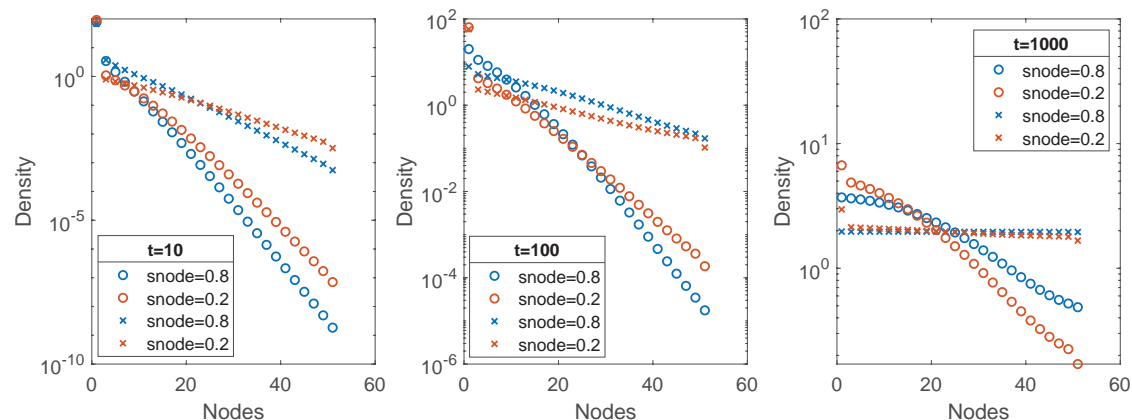

Fig. 10 Density distribution for small nodes $\Omega_{s}, s_{\text {net }}=0.8(\circ)$, resp., $0.4(\times)$.
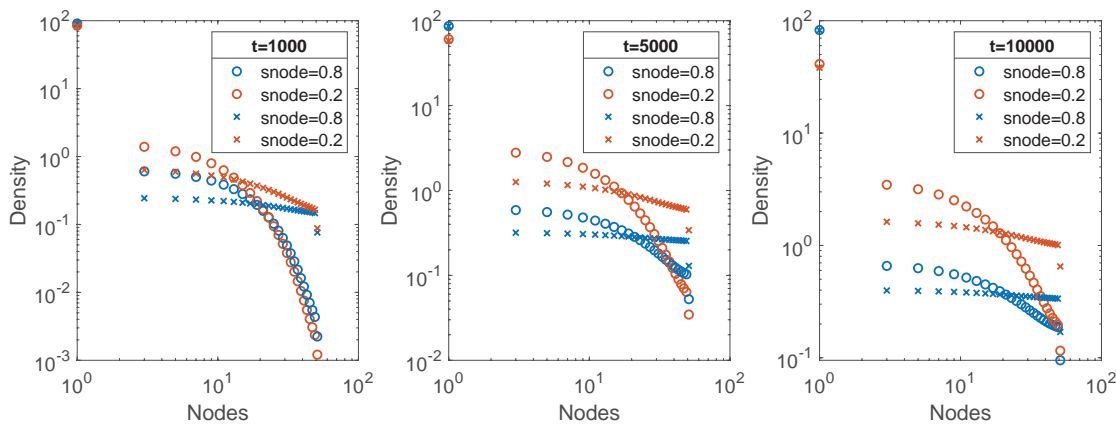

Fig. II Density distribution for big nodes $\Omega_{b}, s_{n e t}=0.8(\circ)$, resp., $0.4(\times)$.

risk of moving rapidly away from the target due to the nature of their Lévy walk. Individuals with lower motility were slower but more precise.

The following figures compare these conclusions to those obtained for a network of big nodes $\Omega_{b}$.

In Figure 9(b) we observe that superdiffusion inside big nodes speeds up the equilibration of the densities considerably: For $s_{\text {nod }}=0.2$ particles require a much 
smaller time to reach their distant target, the sink, than for $s_{n o d}=0.8$. This is in agreement with the discussion in subsection 4.1.

In Figure 11 we plot the density as a function of the node, in a log-log plot at times $t=1000,5000,10000$. Note that because of the large distance between sources and sinks for nodes $\Omega_{b}$, the time scale to approach equilibrium increases significantly. We observe that superdiffusion inside the nodes accelerates the equilibration over the whole metaplex in all cases, unlike for the small nodes $\Omega_{s}$ (Figure 10). For $s_{n e t}=0.4$ in Figure 11 we observe the accelerated diffusion clearly, with a density distribution that is far from a Gaussian parabola in the log-log plot.

6.3.I. Metaplex Superdiffusion. From the discussion of Experiments 1 and 2 so far, we conclude that for the short range network diffusion (2.7), proportional to $2^{-s_{n e t}|i-j|}$, superdiffusion in the nodes can accelerate the equilibration in the metaplex, but it cannot lead to global superdiffusion. This is in line with the discussion in section 5 . We now consider a long range network coupling proportional to $|i-j|^{-s_{n e t}}$ given by the Mellin-transformed $d$-path Laplacian (2.6) for $s_{n e t}=1.5,2,2.5$, and 4 .

In the case of this linear metaplex we count on the advantage that previous analytic results exist for the diffusion on its exoskeleton using the $d$-path Laplacians [19]. In this case it was proved analytically that the use of the Mellin transform may produce a superdiffusive regime in an infinite linear chain of nodes for $s_{\text {net }} \in(1,3)$.

As in Figure 11, Figure 12 plots the density at times $t=10,100,1000,10000$ as a function of the node of the network in a log-log plot. The linear decay of the density and the peaked behavior at node 1 indicate superdiffusion irrespective of the internal dynamics. Because of the strong network diffusion, for the big nodes $\Omega_{b}$ the structure of the nodes proves irrelevant to the metaplex dynamics.

In Figure 13 we exhibit the absence of superdiffusion for large Mellin exponents $s_{\text {net }}=4$ and $s_{\text {nod }}=0.8$ : The density distribution recovers a Gaussian shape, characteristic of normal diffusion, as is clearly visible for longer times. Note that for the illustration of the Gaussian shape we have symmetrically reflected the network with respect to the $y$-axis.

Finally, Figure 14 shows the equilibration of the density in node 1 with time. It confirms the interplay of the nodal diffusion process and the size of the node, as is observed for the short range network diffusion in Figure 9 above.

In closing, after all the analyses carried out in this section we can conclude that for the path graph $\mathcal{Q}$ and for $T_{i j}$ given by the $d$-path Laplacian (2.7), there exists $C>0$ such that $\int_{\Omega_{j}} u_{j}(t, x) d x \leq C e^{-C|j-1|}$ for $j \in \mathcal{Q}$. In particular, superdiffusion is not possible for the considered internal diffusion $\left\{H_{k}\right\}_{k}$.

In the case of the Mellin-transformed $d$-Laplacian (2.6), the numerical results indicate that superdiffusion in the network may persist even for nodes with distant sinks and sources. While, in general, the range of Mellin exponents $s_{\text {net }}$ that allow superdiffusion may shrink, for certain experiments in the linear network $V=\mathcal{Q}$ we numerically recover superdiffusion for $s_{n e t} \in(1,3)$, as in the absence of internal structure [22].

7. Real-World Metaplexes. In this section we study diffusion in two real-world metaplexes of very distinctive types. The first, a landscape metaplex representing a fragmented forest from the south of Madagascar, shares some of the large-world properties of the linear metaplex in section 6 . A metaplex representing the cortical region of a macaque, used as a second example, illustrates the dominance of the endodynamics in an ultra small-world network. 

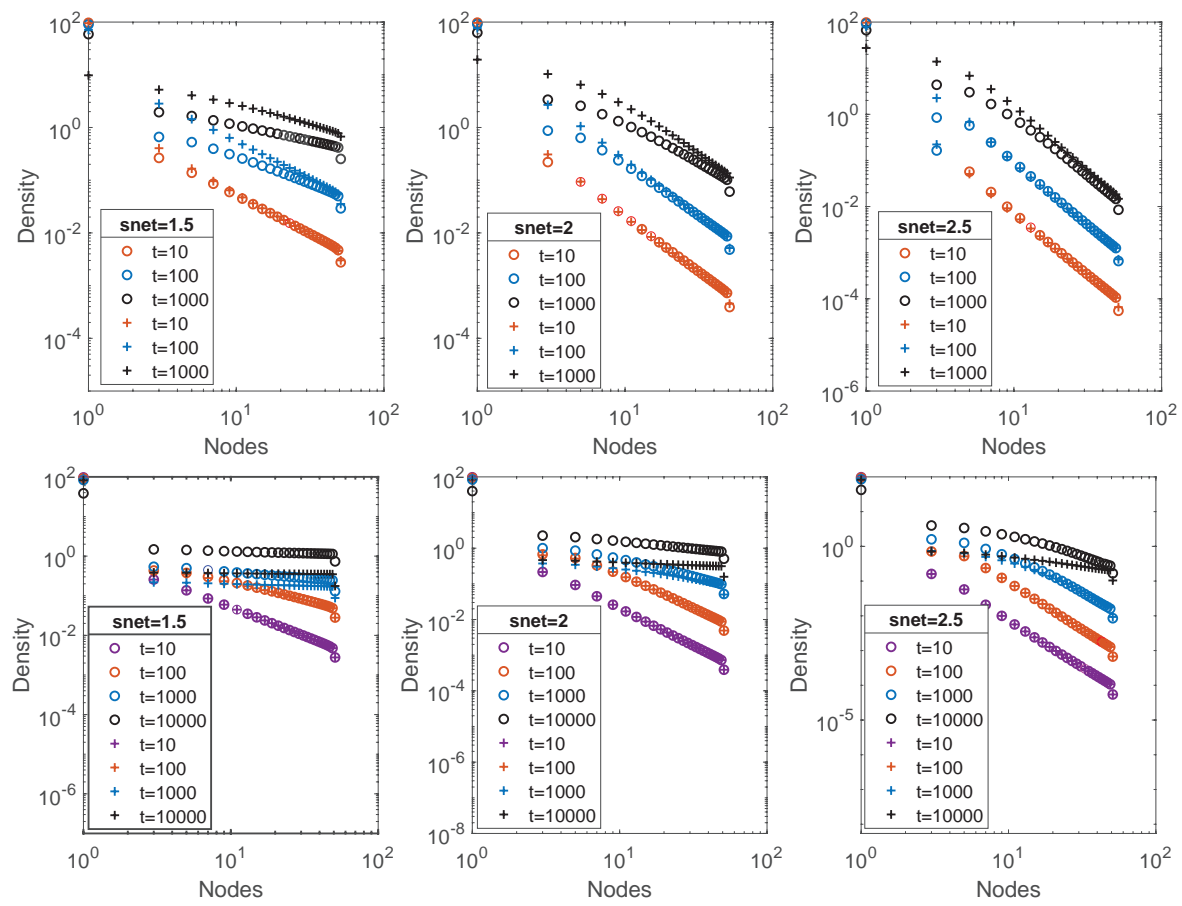

Fig. 12 Density distribution for long range network coupling, $s_{\text {nod }}=0.2(\circ)$ and $s_{\text {nod }}=0.8(+)$. Top panel: $\Omega_{s}$. Bottom panel: $\Omega_{b}$

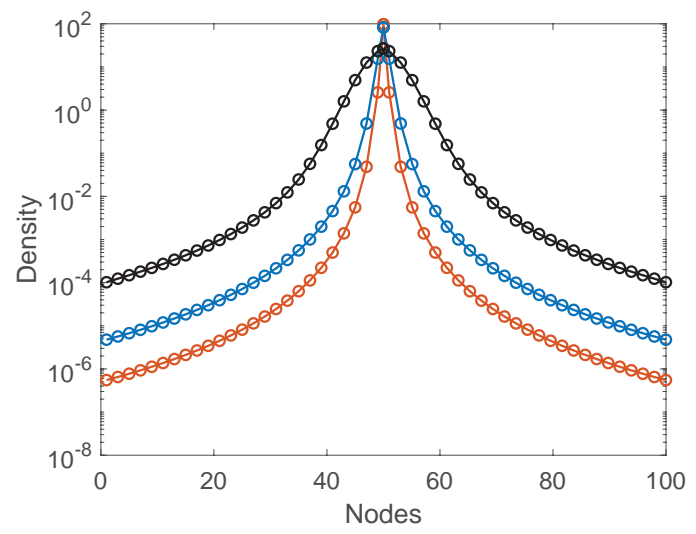

Fig. 13 Gaussian shape of density distribution for $s_{\text {net }}=4$ and $s_{\text {nod }}=0.8$ at $t=10$ (red), $t=100$ (blue), and $t=1000$ (black).

Here we focus on the diffusive processes on both systems. The analogies between brain and ecological anomalous diffusion seem to be more than casual. Costa et al. [12] have coined the term "foraging brain" to refer to these similarities, and the connection between animal foraging and cognitive foraging was considered by Hills [33] on an evolutionary basis. 


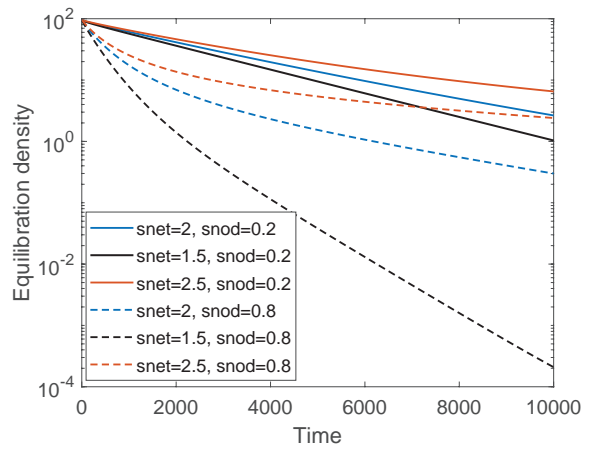

(a)

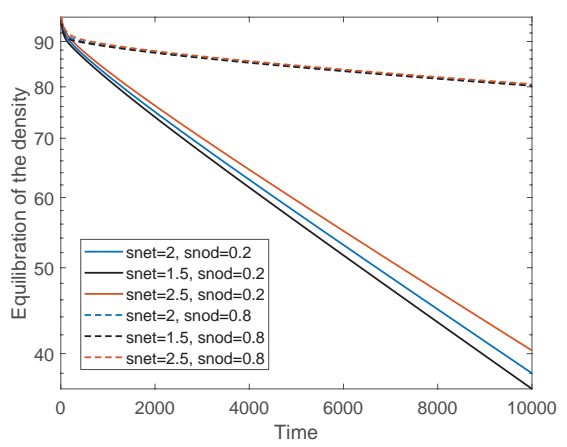

(b)

Fig. 14 Density equilibration in node 1 for the Mellin-transformed d-path Laplacian for $\Omega_{s}$ (a) and $\Omega_{b}(\mathrm{~b})$.

In the following subsections we consider either small $\Omega_{s}$ or big $\Omega_{b}$ disks for all the nodes in the metaplex, exactly as defined for the toy model. The goal of this is to analyze whether the size of the nodes has any influence on the global dynamics of the metaplex. Thus, we can consider that $\Omega_{s}$ and $\Omega_{b}$ are average sizes of the regions in the corresponding metaplex, and such an average is either relatively small or relatively big. The coupling strength is given by $\alpha=10$ for the small nodes $\Omega_{s}$ and $\alpha=100$ for the big nodes $\Omega_{b}$. The nature of the coupling, as for the toy model in section 6 , is considered to be long range, proportional to $\operatorname{dist}(i, j)^{-s_{\text {net }}}$ according to the Mellin transformed $d$-path Laplacian (2.6). The results for the short range coupling can be found in the supplementary material (Note 4).

For the landscape metaplex we discretize each node using a finite element method with 95 degrees of freedom, while for the metaplex of the cortical regions of a macaque we use a mesh of 347 degrees of freedom

The study of real-world metaplexes imposes some limits on the models in use which were not needed for the idealistic toy model studied above. The first is that the consideration of a unique source and sink at the center of each node seems unreasonable. For both cases, the landscape and the visual cortex, we consider spatial regions that can be connected to each other by disjoint sources and sinks as in Figure 5(b).

7.I. Landscape Metaplex. The first example considered represents a landscape in the south of Madagascar that has been fragmented into 183 patches, corresponding to the nodes of a metaplex, as a result of agricultural activity [6]. These patches are connected by 529 narrow corridors that create the exostructure of the metaplex. Such patches are vital for the survival of the ecosystem because they are now the main habitat for the Lemur catta, which plays a fundamental role as a major seed propagator in this environment [5, 21].

Here we assume that all the patches have the same geometry. The network has relatively low edge density - the number of edges divided by the maximum possible number of edges - $\delta \approx 0.032$ with about $45 \%$ of nodes having degrees 2 and 3 , and only two nodes having degree 16 . The average separation between two patches in terms of the shortest path distance is approximately 11.88 with at least one pair of patches separated at $d_{\max }=32$. The average degree of the patches is approximately 5.78 and the maximum number of nearest neighbors that a patch has is 16 . 


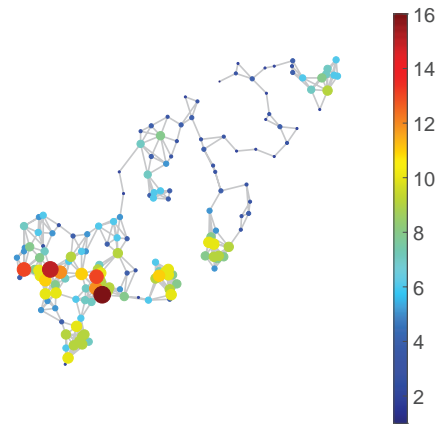

(a)

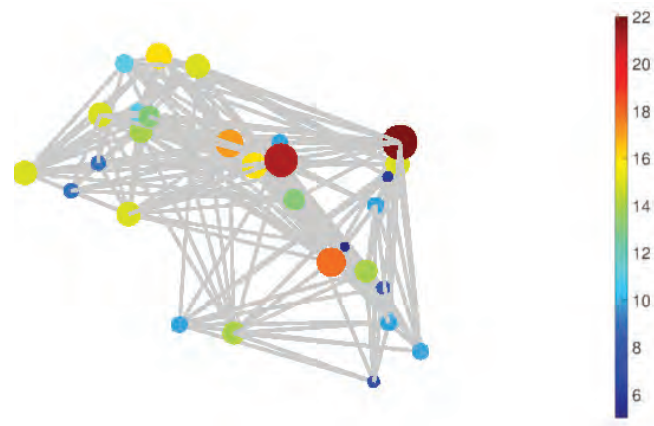

(b)

Fig. I5 Illustration of the exoskeleton of the landscape (a) and the macaque metaplex (b) studied here. The nodes are drawn with size and color proportional to their degree.

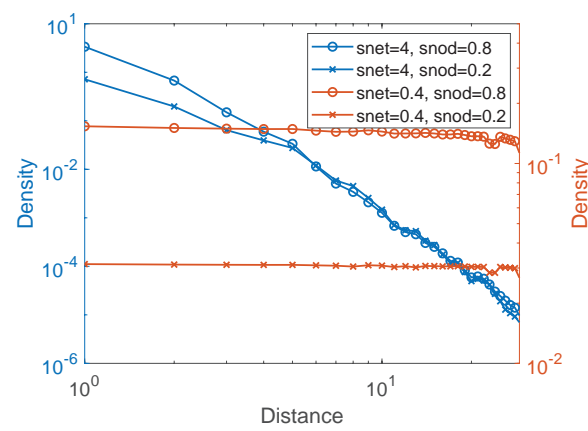

(a)

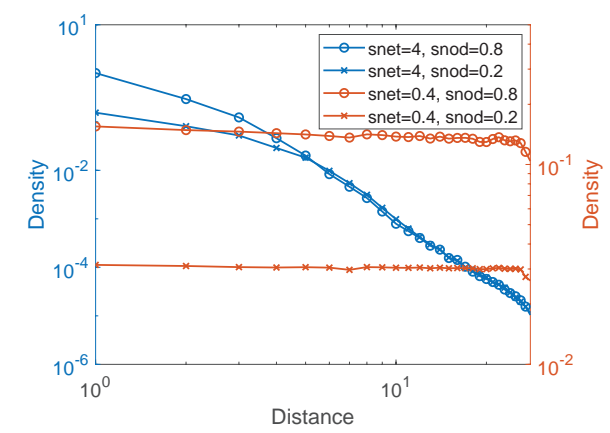

(b)

Fig. 16 Density as a function of distance for disjoint coupling point at $t=100$. (a) Low connectivity nodes. (b) High connectivity nodes.

Also, we can observe that most of the hubs - high degree nodes - are clumped together in a certain region of the landscape (see Figure 15(a)), while the low degree nodes are relatively spread out across the landscape. Consequently, we have initialized the diffusive process by placing the initial condition either into a randomly selected hub or into a randomly selected low degree node.

The next experiment allows us to investigate whether the degree of the node at which the diffusion starts has an important influence on the global metaplex diffusion.

In Figure 16 we plot the density of the diffusive particle as a function of the shortest path distance from the initial condition when the diffusion starts at a low (Figure 16(a)) or at a high (Figure 16(b)) degree node. Here we averaged all the densities for nodes at exactly the same shortest path distance from the initial condition, where three different nodes with high (resp., low) connectivity were chosen to initialize the process. We observe that there are no significant differences between Figure 16(a) and Figure 16(b): Only when $s_{\text {net }}=4$ are there any differences between the processes initiated at low and at high degree nodes. In this particular case, it seems that when the process starts at a low degree node the density at nodes close to the initial condition shares more concentration than those more distant from it. 


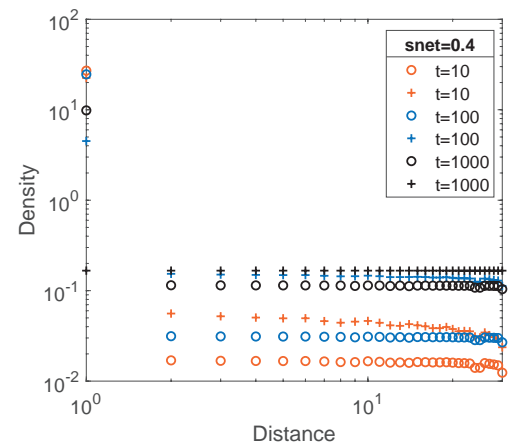

(a)

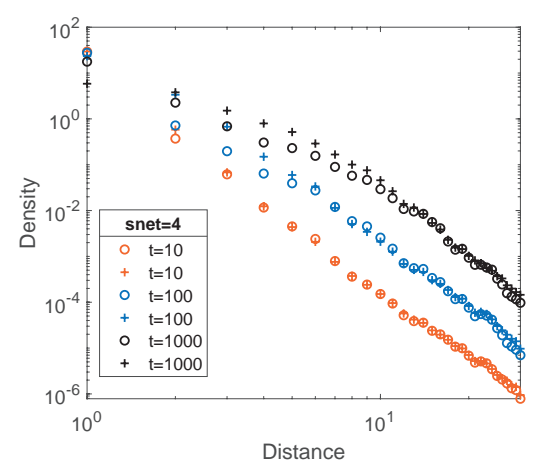

(c)

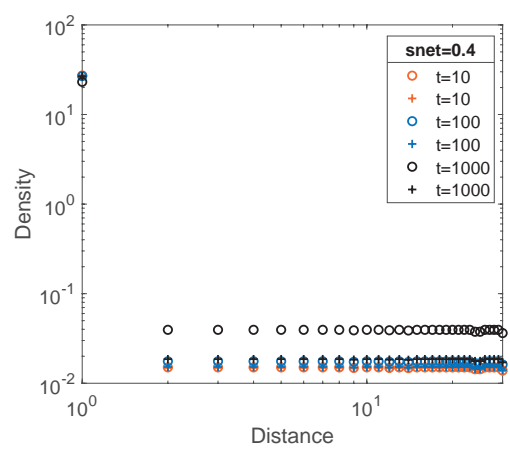

(b)

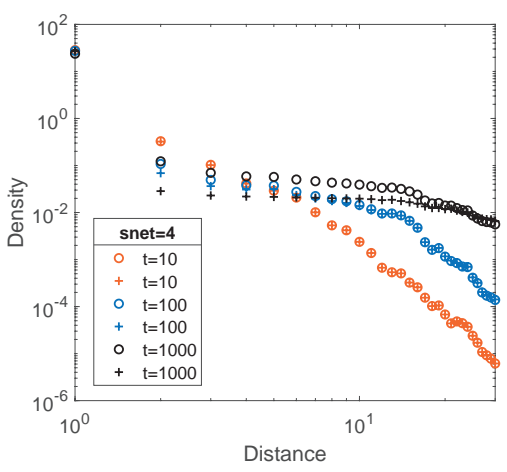

(d)

Fig. 17 Density as a function of distance for disjoint coupling points and snod $=0.8(+)$, snod $=0.2$ (o). (a) and (c) correspond to $\Omega_{s}$ and (b) and (d) correspond to $\Omega_{b}$.

This localization effect can be the consequence not of the degree of the node but of the relative geographic isolation that such nodes can display in this landscape; that is, one low degree node is essentially surrounded by other low degree nodes (see Figure 15(a)). However, in all the other cases it is important to note that the profiles of the density at different distances from the initial conditions are almost exactly the same for processes starting at low or high degree nodes. Consequently, we conclude that in this metaplex the place at which the diffusion is initiated is not relevant for the evolution of the global process. Therefore, in the rest of this section we discuss the results for the process started from low degree nodes.

We now explore the influence of the geometry of the nodes. In Figure 17 we plot the change in the density for nodes at different shortest path distances from the initial condition, for a small (Figure 17(a)) and a big node (Figure 17(b)) using the same value of $s_{n e t}=0.4$. The first clear observation from these plots is that when the nodes are small, superdiffusion inside the nodes slows down the rate to the equilibration of the diffusion in the metaplex (notice that crosses are over the circles in Figure 17(a)). However, when the nodes are big, the reverse occurs, and relatively high values of $s_{\text {nod }}=0.8$ slow down the equilibration of the diffusion in the metaplex (the circles are over the crosses in Figure 17(b).) The same results are reproduced when a much bigger value of $s_{n e t}=4$ is used, as can be seen in Figures 17(c) and 17(d). 

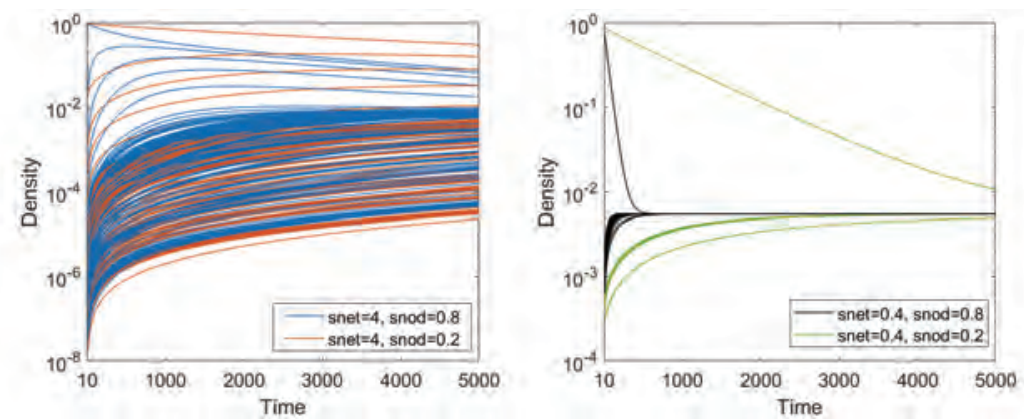

(a)
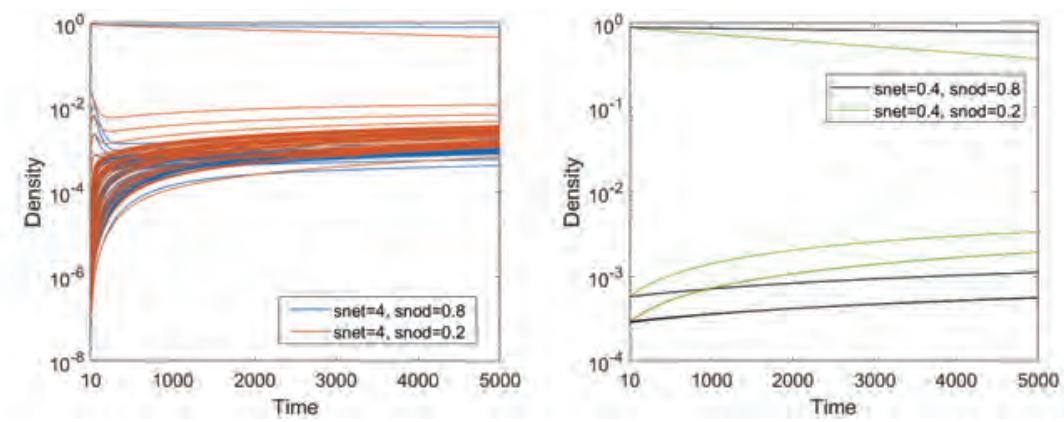

(b)

Fig. 18 Time evolution of the diffusion dynamics for $\Omega_{s}$ (a) and $\Omega_{b}$ (b) with disjoint sinks and sources inside the node of the landscape metaplex.

The reason for this apparently counterintuitive behavior has been previously discussed for the toy model: in a small node it is counterproductive to make long jumps inside the node, since the diffusive particle will rarely find the sink to escape from the node. In a similar fashion, inside a big node it is counterproductive to make small jumps as that will slow down the mobility of particles away from the source to reach the sink and leave the node. We again conclude that the size of the nodes influences the global dynamics of the metaplex. Also, importantly, the nature of the internal structure can significantly change the rate of convergence of the process and can make it faster or slower depending on the size of the nodes and the nature of the diffusive processes inside them.

Let us now consider the combined influence of the endo- and exodynamics on the global metaplex diffusion. We start by considering the time evolution of the diffusive particle across the metaplex after initiating the process at a randomly selected node.

In Figure 18(a) we illustrate the time evolution of the density (in logarithmic scale) of the diffusive particle. In the left panel of Figure 18(a) we observe that the diffusion with $s_{\text {nod }}=0.8$ converges to the steady state at an earlier time than that for $s_{\text {nod }}=0.2$ when the same value of $s_{\text {net }}=4$ is used. The same is observed, even more clearly, in the right panel where the process for $s_{\text {nod }}=0.8$ and $s_{\text {net }}=0.4$ converges significantly faster to the steady state than that for $s_{\text {nod }}=0.2$ and $s_{\text {net }}=0.4$. See Table 1 for the values of the standard deviation introduced in section 2 .

Making the nodes bigger shows the same behavior as is discussed above. The slower dynamics, compared to the small nodes (see Figure 18(b)), is expected from 
Table I Standard deviation at $t=5000$ for the landscape metaplex.

\begin{tabular}{|c|c|c|c|c|}
\hline & \multicolumn{2}{|c|}{$s_{\text {net }}=4$} & \multicolumn{2}{c|}{$s_{\text {net }}=0.4$} \\
\hline & $s_{\text {nod }}=0.2$ & $s_{\text {nod }}=0.8$ & $s_{\text {nod }}=0.2$ & $s_{\text {nod }}=0.8$ \\
\hline$\Omega_{s}$ & 0.0265 & 0.0088 & $3.9 \cdot 10^{-4}$ & $1.8 \cdot 10^{-11}$ \\
\hline$\Omega_{b}$ & 0.034 & 0.059 & 0.028 & 0.059 \\
\hline
\end{tabular}

the fact that now the diffusive particle has more area to diffuse in before finding its way out of the node. However, the most interesting observation is that now, as we have previously pointed out, the order of convergence rates is reversed. For the bigger node, the global diffusion equilibrates faster when we have superdiffusion inside the nodes than when $s_{\text {nod }}=0.8$ (compare values in Table 1 for $\Omega_{b}$ ). Similar results are observed for the case $s_{\text {net }}=0.4$.

From Figure 18(a) we observe that varying $s_{n e t}$ and $s_{\text {nod }}$ changes the rate of convergence of the diffusion in the metaplex. So we conclude that, although the landscape metaplex has a large-world exoskeleton, it displays a trade-off between the exo- and endodynamics that determines the global convergence of the diffusion in the metaplex. It is neither the exostructure nor the endostructure alone, but a complex interrelation of both that controls the global dynamics in the metaplex.

7.2. Visual Cortex Metaplex of Macaque. The second metaplex consists of 30 regions of the macaque visual cortex. These cortex regions are functionally connected by 190 edges, which makes the exostructure of this system very dense, with density $\delta \approx 0.437$. As a consequence of this high edge density the network is an "ultrasmallworld" with average shortest path distance between cortex regions of $\bar{d} \approx 1.54$ and a maximum separation between two of these regions of only $d_{\max }=3$ steps. This makes the exoskeleton of this metaplex an "ultra small-world." The average degree is approximately 12.67 with a maximum number of connections at a given node is 22 .

This network displays an almost uniform degree distribution, particularly for degrees between 4 and 16 . Therefore, we just pick at random the nodes to initiate the diffusion on the metaplex since most of the nodes are degree-equivalent. We then proceed with a similar analysis for the case of the landscape metaplex by studying the effects of the endo- and exodynamics on the global diffusion.

When the nodes are small as in Figure 19(a), we observe the same effect as in the previous cases, namely, that larger values of $s_{\text {nod }}$ favor the convergence of the dynamics to the steady state (see Table 2 for the standard deviation values). The results for the big nodes (Figure 19(b)) are similar to those for the toy model and landscape metaplex, i.e., superdiffusion inside the nodes favors the convergence of the dynamics to the steady state.

For the macaque visual cortex metaplex the change of $s_{\text {net }}$ changes practically nothing in the global dynamics. For instance, we see from Figure 19(a) that keeping constant $s_{\text {nod }}=0.8$ and dropping $s_{\text {net }}$ from 0.8 (blue lines in the left panel) to $s_{\text {net }}=0.4$ (black lines in the right panel) leaves the time evolution of the diffusion almost unaffected. The standard deviations at $t=5000$ time units are in Table 2 . We observe similar behavior for the case of big nodes (see Figure 19(b)).

These results are in clear contrast with the ones previously discussed for the landscape metaplex. In the previous case it was observed that a trade-off between the exo- and endodynamics controls the global diffusion at the metaplexic scale. Here, the exodynamics does not play any significant role in the global dynamics of the 

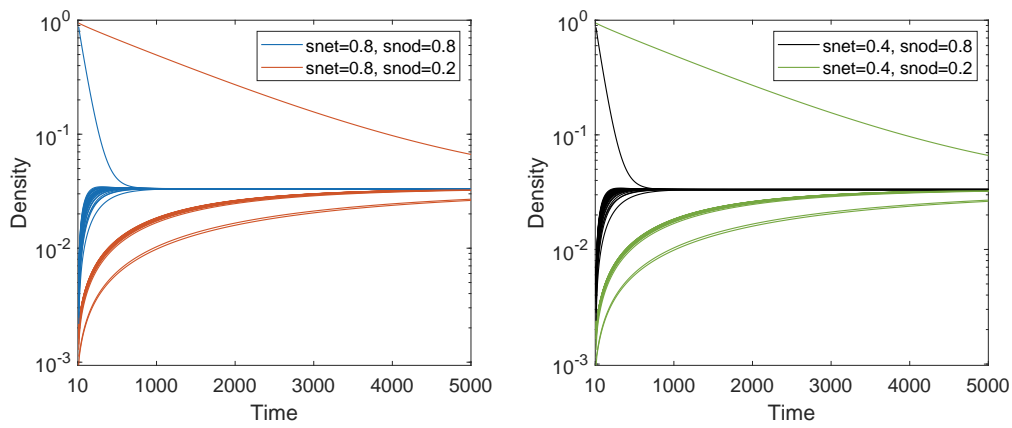

(a)
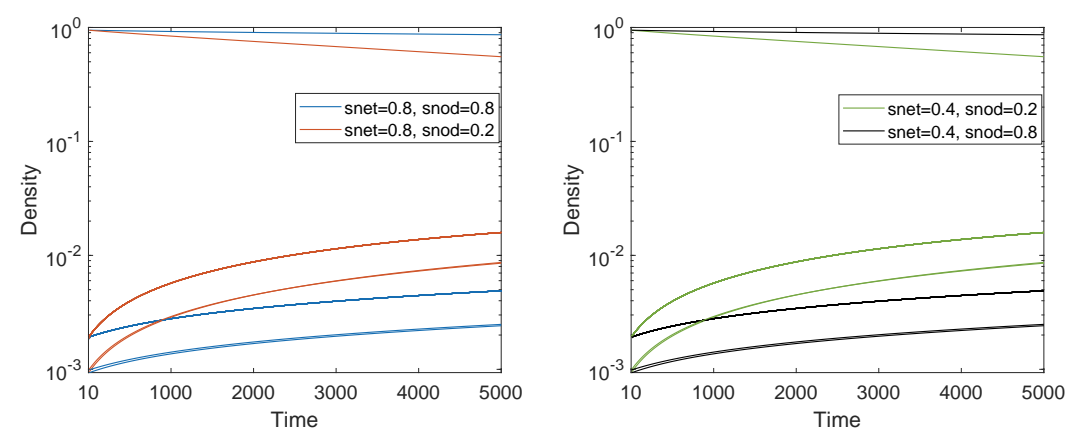

(b)

Fig. 19 Time evolution of the diffusion process on the macaque visual cortex metaplex using different parameters for the endo- and exodynamics (see text for details).

Table 2 Standard deviation at $t=5000$ for the macaque visual cortex metaplex.

\begin{tabular}{|c|c|c|c|c|}
\hline & \multicolumn{2}{|c|}{$s_{\text {net }}=0.8$} & \multicolumn{2}{c|}{$s_{\text {net }}=0.4$} \\
\hline & $s_{\text {nod }}=0.2$ & $s_{\text {nod }}=0.8$ & $s_{\text {nod }}=0.2$ & $s_{\text {nod }}=0.8$ \\
\hline$\Omega_{s}$ & 0.0064 & $1.378 \cdot 10^{-9}$ & 0.0064 & $1.3283 \cdot 10^{-9}$ \\
\hline$\Omega_{b}$ & 0.0983 & 0.1567 & 0.0983 & 0.1567 \\
\hline
\end{tabular}

metaplex. The factor which determines such global dynamics is the endostructure of the nodes in the metaplex. The reasons for this extreme case of dependence of the global dynamics almost exclusively is two-fold. First, the exoskeleton of the macaque visual cortex analyzed here is an ultra small-world, where long range hops are not possible. The second is the almost-complete nature of this exostructure, i.e., its high density and uniformity of degree distribution. It is intuitively clear that in a complete network there are no topological effects that can affect the dynamics. In this case only the endodynamics has a significant influence on the metaplexic global dynamics.

Most real-world metaplexes are expected to have exostructures that are not as dense and uniform as that of the macaque visual cortex. Although they certainly display small-world properties, they show a larger variability of shortest path distances than the macaque cortex. Thus, it is expected that they display certain trade-offs between the endo- and the exostructures such as that observed here for the landscape metaplex in determining the global dynamics of the system. 
8. Summary. The concept of metaplex introduced in this work allows us to study the trade-off between the internal structure and dynamics of/in the nodes. Here we provide the basic notions and motivations for the study of metaplexes with continuous internal structure of the nodes and discrete internode dynamics. Additionally, we provide theoretical and computational support for a series of results related to the study of diffusive dynamics on metaplexes. In particular, we present examples in which the endostructure of the metaplex determines almost uniquely the global dynamics: in the macaque visual cortex the exostructure plays no fundamental role. On the other hand, in the linear metaplex chain there is a trade-off between the endo- and exodynamics to determine the global diffusion. This is reflected in the Madagascar metaplex landscape. We have also studied here the effect of the geometry, such as the size of nodes, location of sinks and sources, and the nature and strength of the coupling between nodes.

Our numerical results show that superdiffusion due to long range hopping in the linear network $\mathcal{Q}=\{\ldots,-2,-1,0,1,2, \ldots\}$, as in [22], survives irrespective of the internal structure of the nodes (Figure 12). The parameter range of Mellin exponents for which superdiffusion is observed, $s_{n e t} \in(1,3)$, is the same as for ordinary networks of point nodes without internal structure. The conclusion extends to certain networks from applications of a large diameter, here studied for the landscape network of habitats of $L$. catta (Figure 17). The combined influence of the endo- and exostructures determines the global diffusion in such networks.

The endostructure, on the other hand, dominates at shorter distances and therefore becomes crucial for diffusion in small-world metaplexes. This was illustrated for the cortical metaplex of the macaque in Figure 19. The effect of the internal dynamics in the whole metaplex depends strongly on the geometry of the nodes and the nature of the coupling. When sinks and sources overlap, internal superdiffusion may slow down the metaplex dynamics, and normal diffusion is faster on small scales. When sinks and sources are in separate, distant locations, superdiffusion in the nodes allows particles to explore the entire metaplex much faster than classical diffusion. While it accelerates diffusion, the internal superdiffusion cannot, by itself, induce superdiffusion in the metaplex.

Our results can be understood from the local description we provide of the distribution of particles inside each node. The combination of analytical methods for the PDE description in the node, and network methods for their interconnection in the exoskeleton, gives a new perspective not only on classical complex network descriptions, but also on the study of PDEs describing physical systems which can be either split into continuous regions interconnected in a discrete way or which involve a network of internal degrees of freedom. This illustrates how the study of metaplexes draws tools from a wide range of areas, such as the geometric analysis of coupled PDEs and interface problems, spectral theory of operator matrices, high-dimensional stochastic processes, etc. Conversely, it suggests the relevance of network theory for old problems in these fields, such as the problem of finding effective descriptions of interacting many-particle systems with high-dimensional internal degrees of freedom.

From a computational point of view, numerical experiments for large metaplexes become a challenge, due to the new internal degrees of freedom in each node. For applications to real-world networks, future work should explore methods that represent this internal dynamics efficiently. Model order reduction or generalized finite element methods [52] are examples that have been used already in related settings to achieve a reasonable accuracy for small degrees of freedom. 
REFERENCES

[1] F. Bartumeus, P. Fernández, M. da Luz, J. Catalan, R. V. Solé, and S. A. Levin, Superdiffusion and encounter rates in diluted, low dimensional worlds, European Phys. J. Special Topics, 157 (2008), pp. 157-166. (Cited on p. 620)

[2] J. Bertoin, Lévy Processes, Cambridge University Press, 1998. (Cited on p. 629)

[3] S. Boccaletti, G. Bianconi, R. Criado, C. I. Del Genio, J. Gómez-Gardenes, M. Romance, I. Sendia-NAdal, Z. WANG, And M. Zanin, The structure and dynamics of multilayer networks, Phys. Rep., 544 (2014), pp. 1-122. (Cited on p. 619)

[4] S. Boccaletti, V. Latora, Y. Moreno, M. Chávez, and D.-U. Hwang, Complex networks: Structure and dynamics, Phys. Rep., 424 (2006), pp. 175-308. (Cited on p. 618)

[5] Ö. Bodin AND J. NoRBERG, A network approach for analyzing spatially structured populations in a fragmented landscape, Landscape Ecol., 22 (2007), pp. 31-44. (Cited on p. 636)

[6] Ö. Bodin, M. Tengö, A. Norman, J. LundberG, And T. Elmqvist, The value of small size: Loss of forest patches and ecological thresholds in southern Madagascar, Ecological Appl., 16 (2006), pp. 440-451. (Cited on p. 636)

[7] J.-P. Bouchaud And A. Georges, Anomalous diffusion in disordered media: Statistical mechanisms, models and physical applications, Phys. Rep., 195 (1990), pp. 127-293. (Cited on p. 620$)$

[8] E. Bullmore and O. Sporns, Complex brain networks: Graph theoretical analysis of structural and functional systems, Nature Rev. Neurosci., 10 (2009), p. 186. (Cited on pp. 618, 620)

[9] J. Burrow, P. BaXter, AND J. PitchFord, Lévy processes, saltatory foraging, and superdiffusion, Math. Model. Nat. Phenom., 3 (2008), pp. 115-130. (Cited on p. 620)

[10] X. Chen, L. Guo, J. Kang, Y. Huo, S. Wang, and W. Tan, Calcium waves initiating from the anomalous subdiffusive calcium sparks, J. Roy. Soc. Interface, 11 (2014), art. 20130934. (Cited on p. 620)

[11] A. Cornell-Bell and S. Finkbeiner, Ca2+ waves in astrocytes, Cell Calcium, 12 (1991), pp. 185-204. (Cited on p. 620)

[12] T. Costa, G. Boccignone, F. CAuda, and M. Ferraro, The foraging brain: Evidence of Levy dynamics in brain networks, PloS One, 11 (2016), art. e0161702. (Cited on pp. 620, $635)$

[13] R. Courant and D. Hilbert, Methods of Mathematical Physics: Partial Differential Equations, John Wiley \& Sons, 2008. (Cited on p. 626)

[14] S. Dipierro, X. Ros-Oton, and E. Valdinoci, Nonlocal problems with Neumann boundary conditions, Rev. Mat. Iberoam., 33 (2017), pp. 377-416. (Cited on p. 622)

[15] J. F. Donges, Y. Zou, N. Marwan, and J. Kurths, Complex networks in climate dynamics, European Phys. J. Special Topics, 174 (2009), pp. 157-179. (Cited on pp. 619, 620)

[16] M. Endo, M. TANAKA, And Y. OGaWA, Calcium induced release of calcium from the sarcoplasmic reticulum of skinned skeletal muscle fibres, Nature, 228 (1970), p. 34. (Cited on p. 620)

[17] R. ERban AND H. G. OthmeR, From individual to collective behavior in bacterial chemotaxis, SIAM J. Appl. Math., 65 (2004), pp. 361-391, https://doi.org/10.1137/ S0036139903433232. (Cited on p. 625)

[18] R. Erban and H. G. Othmer, From signal transduction to spatial pattern formation in e. coli: A paradigm for multiscale modeling in biology, Multiscale Model. Simul., 3 (2005), pp. $362-$ 394, https://doi.org/10.1137/040603565. (Cited on p. 625)

[19] E. Estrada, Path Laplacian matrices: Introduction and application to the analysis of consensus in networks, Linear Algebra Appl., 436 (2012), pp. 3373-3391. (Cited on pp. 622, 634)

[20] E. Estrada, The Structure of Complex Networks: Theory and Applications, Oxford University Press, 2012. (Cited on p. 618)

[21] E. Estrada AND Ö. Bodin, Using network centrality measures to manage landscape connectivity, Ecological Appl., 18 (2008), pp. 1810-1825. (Cited on p. 636)

[22] E. Estrada, E. Hameed, N. Hatano, And M. Langer, Path Laplacian operators and superdiffusive processes on graphs. I. One-dimensional case, Linear Algebra Appl., 523 (2017), pp. 307-334. (Cited on pp. 620, 623, 634, 642)

[23] E. Estrada, E. Hameed, M. Langer, and A. Puchalska, Path Laplacian operators and superdiffusive processes on graphs. II. Two-dimensional lattice, Linear Algebra Appl., 555 (2018), pp. 373-397. (Cited on p. 623)

[24] G. Estrada-Rodriguez, H. Gimperlein, K. J. Painter, and J. Stocek, Space-time fractional diffusion in cell movement models with delay, Math. Models Methods Appl. Sci., 29 (2019), pp. 65-88. (Cited on pp. 627, 630) 
[25] A. Fabiato and F. Fabiato, Excitation-contraction coupling of isolated cardiac fibers with disrupted or closed sarcolemmas: Calcium-dependent cyclic and tonic contractions, Circulation Res., 31 (1972), pp. 293-307. (Cited on p. 620)

[26] E. Frey and K. Kroy, Brownian motion: A paradigm of soft matter and biological physics, Ann. Phys., 14 (2005), pp. 20-50. (Cited on p. 619)

[27] J. Gao, S. V. Buldyrev, S. Havlin, And H. E. Stanley, Robustness of a network of networks, Phys. Rev. Lett., 107 (2011), art. 195701. (Cited on p. 619)

[28] H. Gimperlein AND G. GRuBb, Heat kernel estimates for pseudodifferential operators, fractional Laplacians and Dirichlet-to-Neumann operators, J. Evol. Eq., 14 (2014), pp. 49-83, https://doi.org/10.1007/s00028-013-0206-2. (Cited on p. 629)

[29] I. Golding And E. C. Cox, Physical nature of bacterial cytoplasm, Phys. Rev. Lett., 96 (2006), art. 098102. (Cited on p. 620)

[30] S. Gómez, A. Diaz-Guilera, J. Gómez-Gardenes, C. J. Pérez-Vicente, Y. Moreno, and A. Arenas, Diffusion dynamics on multiplex networks, Phys. Rev. Lett., 110 (2013), art. 028701. (Cited on p. 619)

[31] J. Goñi, G. Arrondo, J. Sepulcre, I. Martincorena, N. V. de Mendizábal, B. Corominas-Murtra, B. Bejarano, S. Ardanza-Trevijano, H. Peraita, D. P. Wall, and P. VILlOSLADA, The semantic organization of the animal category: Evidence from semantic verbal fluency and network theory, Cognitive Process., 12 (2011), pp. 183-196. (Cited on pp. 618,619$)$

[32] A. Grigor'yan, Heat kernels and function theory on metric measure spaces, in Heat Kernels and Analysis on Manifolds, Graphs, and Metric Spaces (Paris, 2002), Contemp. Math. 338, AMS, 2003, pp. 143-172. (Cited on p. 628)

[33] T. T. HiLls, Animal foraging and the evolution of goal-directed cognition, Cognitive Sci., 30 (2006), pp. 3-41. (Cited on p. 635)

[34] W. Jo, A. LAw, And S. Chung, The neglected co-star in the dementia drama: The putative roles of astrocytes in the pathogeneses of major neurocognitive disorders, Molecular Psych., 19 (2014), p. 159. (Cited on p. 620)

[35] B. S. Kenner, The Physics of Traffic: Empirical Freeway Pattern Features, Engineering Applications, and Theory, Springer, 2012. (Cited on p. 619)

[36] M. Kivelä, A. Arenas, M. Barthelemy, J. P. Gleeson, Y. Moreno, and M. A. Porter, Multilayer networks, J. Complex Networks, 2 (2014), pp. 203-271. (Cited on p. 619)

[37] M. Köpf, C. Corinth, O. Haferkamp, and T. Nonnenmacher, Anomalous diffusion of water in biological tissues, Biophys. J., 70 (1996), pp. 2950-2958. (Cited on p. 620)

[38] H. A. Kramers, Brownian motion in a field of force and the diffusion model of chemical reactions, Physica, 7 (1940), pp. 284-304. (Cited on p. 619)

[39] E. G. LAKATta AND T. GUARNIERI, Spontaneous myocardial calcium oscillations: Are they linked to ventricular fibrillation?, J. Cardiovascular Electrophysiol., 4 (1993), pp. 473-489. (Cited on p. 620)

[40] V. Latora, V. Nicosia, and G. Russo, Complex Networks: Principles, Methods and Applications, Cambridge University Press, 2017. (Cited on p. 618)

[41] M. Lax, W. CaI, and M. Xu, Random Processes in Physics and Finance, Oxford University Press, 2006. (Cited on p. 619)

[42] L. Lu, L. XIA, X. YE, AND H. Cheng, Simulation of the effect of rogue ryanodine receptors on a calcium wave in ventricular myocytes with heart failure, Phys. Biol., 7 (2010), art. 026005. (Cited on p. 620)

[43] K. LubY-PhelPS, Cytoarchitecture and physical properties of cytoplasm: Volume, viscosity, diffusion, intracellular surface area, Internat. Rev. Cytology, 192 (1999), pp. 189-221. (Cited on p. 619)

[44] F. MatthäUs, M. JAGOdič, AND J. DoBnikar, E. coli superdiffusion and chemotaxis-search strategy, precision, and motility, Biophys. J., 97 (2009), pp. 946-957. (Cited on p. 632)

[45] D. M. McAvity And H. Osborn, A DeWitt expansion of the heat kernel for manifolds with a boundary, Classical Quantum Gravity, 8 (1991), pp. 603-638, http://stacks.iop.org/ 0264-9381/8/i=4/a=008. (Cited on p. 629)

[46] R. Metzler and J. Klafter, The random walk's guide to anomalous diffusion: A fractional dynamics approach, Phys. Rep., 339 (2000), pp. 1-77. (Cited on pp. 620,622)

[47] R. Metzler and J. Klafter, The restaurant at the end of the random walk: Recent developments in the description of anomalous transport by fractional dynamics, J. Phys. A, 37 (2004), pp. R161-R208. (Cited on p. 622)

[48] M. Nedergand, B. Ransom, and S. A. Goldman, New roles for astrocytes: Redefining the functional architecture of the brain, Trends Neurosci., 26 (2003), pp. 523-530. (Cited on p. 618)

Copyright $@$ by SIAM. Unauthorized reproduction of this article is prohibited. 
[49] M. E. J. Newman, The structure and function of complex networks, SIAM Rev., 45 (2003), pp. 167-256, https://doi.org/10.1137/S003614450342480. (Cited on p. 618)

[50] C. Nicholson, Diffusion and related transport mechanisms in brain tissue, Rep. Progr. Phys., 64 (2001), pp. 815-884. (Cited on p. 620)

[51] D. V. Nicolau, Jr., J. F. Hancock, and K. Burrage, Sources of anomalous diffusion on cell membranes: A Monte Carlo study, Biophys. J., 92 (2007), pp. 1975-1987. (Cited on p. 620)

[52] P. O'Hara, C. A. Duarte, And T. EAson, Transient analysis of sharp thermal gradients using coarse finite element meshes, Comput. Methods Appl. Mech. Engrg., 200 (2011), pp. 812-829. (Cited on p. 642)

[53] E.-M. Ouhabaz, Analysis of Heat Equations on Domains, London Math. Soc. 31, Princeton University Press, 2009. (Cited on p. 628)

[54] B. Perthame, W. Sun, And M. TAng, The fractional diffusion limit of a kinetic model with biochemical pathway, Z. Angew. Math. Phys., 69 (2018), art. 67. (Cited on p. 625)

[55] M. A. Porter, What is ... a multilayer network, Notices Amer. Math. Soc., 11 (2018), pp. 1419-1423. (Cited on p. 619)

[56] J. F. Reverey, J.-H. Jeon, H. Bao, M. Leippe, R. Metzler, and C. Selhuber-Unkel, Superdiffusion dominates intracellular particle motion in the supercrowded cytoplasm of pathogenic Acanthamoeba castellanii, Sci. Rep., 5 (2015), art. 11690. (Cited on p. 620)

[57] E. B. Ridgway, J. C. Gilkey, And L. F. JafFe, Free calcium increases explosively in activating medaka eggs, Proc. Natl. Acad. Sci. USA, 74 (1977), pp. 623-627. (Cited on p. 620)

[58] H. Sanabria, Y. Kubota, And M. N. Waxham, Multiple diffusion mechanisms due to nanostructuring in crowded environments, Biophys. J., 92 (2007), pp. 313-322. (Cited on p. 620)

[59] C. Seshadhri, T. G. Kolda, And A. Pinar, Community structure and scale-free collections of Erdös-Rényi graphs, Phys. Rev. E, 85 (2012), art. 056109. (Cited on p. 619)

[60] S. Smith, C. Cianci, And R. Grima, Macromolecular crowding directs the motion of small molecules inside cells, J. Roy. Soc. Interface, 14 (2017), art. 20170047. (Cited on p. 620)

[61] A. Sole-Ribalta, M. De Domenico, N. E. Kouvaris, A. Diaz-Guilera, S. Gómez, and A. Arenas, Spectral properties of the Laplacian of multiplex networks, Phys. Rev. E, 88 (2013), art. 032807. (Cited on p. 627)

[62] C. TRetTer, Spectral Theory of Block Operator Matrices and Applications, World Scientific, 2008. (Cited on p. 625)

[63] D. Urban and T. KeitT, Landscape connectivity: A graph-theoretic perspective, Ecology, 82 (2001), pp. 1205-1218. (Cited on pp. 618, 620)

[64] G. Viswanathan, F. Bartumeus, S. V. Buldyrev, J. Catalan, U. Fulco, S. Havlin, M. Da Luz, M. L. Lyra, E. Raposo, and H. E. Stanley, Lévy flight random searches in biological phenomena, Phys. A, 314 (2002), pp. 208-213. (Cited on p. 620)

[65] S. C. Weber, A. J. Spakowitz, and J. A. Theriot, Bacterial chromosomal loci move subdiffusively through a viscoelastic cytoplasm, Phys. Rev. Lett., 104 (2010), art. 238102, https://doi.org/10.1103/PhysRevLett.104.238102. (Cited on p. 620)

[66] J. Wetherington, G. Serrano, and R. Dingledine, Astrocytes in the epileptic brain, Neuron, 58 (2008), pp. 168-178, https://doi.org/https://doi.org/10.1016/j.neuron.2008.04.002. (Cited on p. 620)

[67] K. Yamasaki, A. Gozolchiani, And S. Havlin, Climate networks around the globe are significantly affected by El Niño, Phys. Rev. Lett., 100 (2008), art. 228501. (Cited on pp. 619, $620)$

[68] D. H. Zanette, Statistical-thermodynamical foundations of anomalous diffusion, Brazilian J. Phys., 29 (1999), pp. 108-124. (Cited on p. 619)

[69] H.-X. Zhou, G. Rivas, And A. P. Minton, Macromolecular crowding and confinement: Biochemical, biophysical, and potential physiological consequences, Ann. Rev. Biophys., 37 (2008), pp. 375-397, https://doi.org/10.1146/annurev.biophys.37.032807.125817. (Cited on p. 618$)$

[70] S. B. Zimmerman AND S. O. Trach, Estimation of macromolecule concentrations and excluded volume effects for the cytoplasm of Escherichia coli, J. Molecular Biol., 222 (1991), pp. 599620, https://doi.org/https://doi.org/10.1016/0022-2836(91)90499-V. (Cited on p. 618)

Copyright (c) by SIAM. Unauthorized reproduction of this article is prohibited. 